\title{
Periodic Point, Endpoint, and Convergence Theorems for Dissipative Set-Valued Dynamic Systems with Generalized Pseudodistances in Cone Uniform and Uniform Spaces
}

\author{
Kazimierz Włodarczyk and Robert Plebaniak \\ Department of Nonlinear Analysis, Faculty of Mathematics and Computer Science, \\ University of Łódż, Banacha 22, 90-238 Łódź, Poland \\ Correspondence should be addressed to Kazimierz Włodarczyk, wlkzxa@math.uni.lodz.pl \\ Received 29 September 2009; Accepted 17 November 2009 \\ Academic Editor: Mohamed A. Khamsi
}

Copyright (c) 2010 K. Włodarczyk and R. Plebaniak. This is an open access article distributed under the Creative Commons Attribution License, which permits unrestricted use, distribution, and reproduction in any medium, provided the original work is properly cited.

\begin{abstract}
In cone uniform and uniform spaces, we introduce the three kinds of dissipative set-valued dynamic systems with generalized pseudodistances and not necessarily lower semicontinuous entropies, we study the convergence of dynamic processes and generalized sequences of iterations of these dissipative dynamic systems, and we establish conditions guaranteeing the existence of periodic points and endpoints of these dissipative dynamic systems and the convergence to these periodic points and endpoints of dynamic processes and generalized sequences of iterations of these dissipative dynamic systems. The paper includes examples.
\end{abstract}

\section{Introduction}

A set-valued dynamic system is defined as a pair $(X, T)$, where $X$ is a certain space and $T$ is a set-valued map $T: X \rightarrow 2^{X}$; in particular, a set-valued dynamic system includes the usual dynamic system where $T$ is a single-valued map. Here $2^{X}$ denotes the family of all nonempty subsets of a space $X$.

Let $(X, T)$ be a dynamic system. By $\operatorname{Fix}(T), \operatorname{Per}(T)$, and $\operatorname{End}(T)$ we denote the sets of all fixed points, periodic points, and endpoints of $T$, respectively, that is, $\operatorname{Fix}(T)=\{w \in X: w \in$ $T(w)\}, \operatorname{Per}(T)=\left\{w \in X: w \in T^{[q]}(w)\right.$ for some $\left.q \in \mathbb{N}\right\}$ and $\operatorname{End}(T)=\{w \in X:\{w\}=T(w)\}$. For each $x \in X$, a sequence $\left(w_{m}: m \in\{0\} \cup \mathbb{N}\right)$ such that

$$
\forall_{m \in\{0\} \cup \mathbb{N}}\left\{w_{m+1} \in T\left(w_{m}\right)\right\}, \quad w_{0}=x,
$$


is called a dynamic process or a trajectory starting at $w_{0}=x$ of the system $(X, T)$ (for details see Aubin and Siegel [1], Aubin and Ekeland [2], and Aubin and Frankowska [3]). For each $x \in X$, a sequence $\left(w_{m}: m \in\{0\} \cup \mathbb{N}\right)$ such that

$$
\forall_{m \in\{0\} \cup \mathbb{N}}\left\{w_{m+1} \in T^{[m+1]}(x)\right\}, \quad w_{0}=x,
$$

$T^{[m]}=T \circ T \circ \cdots \circ T$ ( $m$-times), $m \in \mathbb{N}$, is called a generalized sequence of iterations starting at $w_{0}=x$ of the system $(X, T)$ (for details see Yuan [4, page 557], Tarafdar and Vyborny [5] and Tarafdar and Yuan [6]). Each dynamic process starting from $w_{0}$ is a generalized sequence of iterations starting from $w_{0}$, but the converse may not be true; the set $T^{[m]}\left(w_{0}\right)$ is, in general, bigger than $T\left(w_{m-1}\right)$. If $(X, T)$ is single valued, then, for each $x \in X$, a sequence $\left(w_{m}: m \in\right.$ $\{0\} \cup \mathbb{N}$ ) such that

$$
\forall_{m \in\{0\} \cup \mathbb{N}}\left\{w_{m+1}=T^{[m+1]}(x)\right\}, \quad w_{0}=x,
$$

is called a Picard iteration starting at $w_{0}=x$ of the system $(X, T)$. If $(X, T)$ is single valued, then (1.1)-(1.3) are identical.

The notion of Banach's contraction belongs to the most fundamental mathematical ideas. Caristi [7], Ekeland [8], Aubin and Siegel [1], Yuan [4], and Kirk [9] extended this notion to several directions (dissipative single-valued maps with lower semicontinuous entropies, variational inequlities for lower semicontinuous maps, dissipative set-valued dynamic systems with not necessarily lower semicontinuous entropies, generalized contractions and asymptotic contractions, resp.). It is not our purpose to give a complete list of related papers here.

Racall that if

Let $X$ be a metric space with metric $d$ and let $(X, T)$ be a single-valued dynamic system.

$$
\exists_{\lambda \in[0,1)} \forall_{x, y \in X}\{d(T(x), T(y)) \leqslant \lambda d(x, y)\}
$$

then $(X, T)$ is called a Banach's contraction (Banach [10]). $(X, T)$ is called contractive if $\forall_{x, y \in X}\{0<d(x, y) \Rightarrow d(T(x), T(y))<d(x, y)\}$. If $\exists_{\epsilon>0} \forall_{x, y \in X}\{0<d(x, y)<\epsilon \Rightarrow d(T(x), T(y))<$ $d(x, y)\}$, then $(X, T)$ is called $\epsilon$-contractive (Edelstein [11]). Contractive and $\epsilon$-contractive maps are some modifications of Banach's contractions.

If $(X, T)$ is single valued and

$$
\forall_{x \in X}\{d(x, T(x)) \leqslant \omega(x)-\omega(T(x))\}
$$

for some $\omega: X \rightarrow[0,+\infty)$, then $T$ is called Caristi's map (Caristi [7]). Caristi's maps (1.5) generalize Banach's contractions (1.4) (for details see Kirk and Saliga [12, page 2766]). Banach's contraction principle and Caristi's fixed point theorem are essentially different: in complete metric space, Banach's contraction is continuous, each Picard iteration of this contraction is convergent to a fixed point and this fixed point is unique (Banach [10]) while Caristi's map is not necessarily continuous and if $\omega$ in (1.5) is lower semicontinuous, then each Picard iteration of this map is convergent to a fixed point and this fixed point is not 
necessarily unique (Caristi [7]). Recall that Ekeland's [8] variational principle concerning lower semicontinuous maps and Caristi's fixed point theorem are equivalent.

A map $\omega: X \rightarrow[0,+\infty)$ is called a weak entropy or entropy of a set-valued dynamic system $(X, T)$ if

$$
\forall_{x \in X} \exists_{y \in T(x)}\{d(x, y) \leqslant \omega(x)-\omega(y)\}
$$

or

$$
\forall_{x \in X} \forall_{y \in T(x)}\{d(x, y) \leqslant \omega(x)-\omega(y)\}
$$

respectively, and $(X, T)$ is called weak dissipative or dissipative if it has a weak entropy or an entropy, respectively; here $\omega$ is not necessarily lower semicontinuous. These two kinds of dissipative maps were introduced and studied by Aubin and Siegel [1]. If $(X, T)$ is single valued, then (1.5)-(1.7) are identical.

Various periodic, fixed point, convergence, and invariant set theorems for contractive and $\epsilon$-contractive single-valued and set-valued dynamic systems have been obtained by Edelstein [11], Ding and Nadler [13], and Nadler [14]. Investigations concerning the existence of fixed points and endpoints and convergence of dynamic processes or generalized sequences of iterations to fixed points or endpoints of single-valued and set-valued generalized contractions (Yuan [4], Tarafdar and Yuan [6, 15], Tarafdar and Chowdhury [16], Tarafdar and Vyborny [5]) and dissipative dynamic systems when entropy $\omega$ is not necessarily lower semicontinuous (Aubin and Siegel [1]) have been conducted by a number of authors in different contexts; for example, see Kirk and Saliga [12], Willems [17], Zangwill [18], Justman [19], Maschler and Peleg [20] and Petrussel, Sîntămărian [21].

In this paper, inspired by these results, we introduce in cone uniform and uniform spaces the three kinds of dissipative set-valued dynamic systems with generalized pseudodistances and with not necessarily lower semicontinuous entropies and we present the methods which are useful for establishing general conditions guaranteeing the existence of periodic points and endpoints of these set-valued dynamic systems and conditions that for each starting point the dynamic processes or generalized sequences of iterations converge and the limit is a periodic point or endpoint (see Sections 3-6). The presented definitions and results are more general and different from those given in the literature and are new even for single-valued and set-valued dynamic systems in metric spaces. For details, see Section 7 where examples, remarks, and some comparisons are included. This paper is a continuation of $[22,23]$.

\section{Dissipative Set-Valued Dynamic Systems with Generalized Pseudodistances in Cone Uniform Spaces}

We define a real normed space to be a pair $(L,\|\cdot\|)$, with the understanding that a vector space $L$ over $\mathbb{R}$ carries the topology generated by the metric $(a, b) \rightarrow\|a-b\|, a, b \in L$.

A nonempty closed convex set $H \subset L$ is called a cone in $L$ if it satisfies: (H1) $\forall_{s \in(0, \infty)}\{s H \subset H\} ;(\mathrm{H} 2) H \cap(-H)=\{0\} ;(\mathrm{H} 3) H \neq\{0\}$. 
It is clear that each cone $H \subset L$ defines, by virtue of " $a \preceq_{H} b$ if and only if $b-a \in H$," an order of $L$ under which $L$ is an ordered normed space with cone $H$. We will write $a<_{H} b$ to indicate that $a \leq_{H} b$ but $a \neq b$.

The following terminologies will be much used.

Definition 2.1 (see [22]). Let X be a nonempty set and let $L$ be an ordered normed space with cone $H$.

(i) The family $D=\left\{\mathrm{p}_{\alpha}: \mathrm{X} \times \mathrm{X} \rightarrow \mathrm{L}, \alpha \in \mathcal{A}\right\}$ is said to be a $P$-family of cone pseudometrics on $X(D$-family, for short) if the following three conditions hold:

(D1) $\forall_{\alpha \in \mathcal{A}} \forall_{x, y \in X}\left\{0 \preceq_{H} p_{\alpha}(x, y) \wedge x=y \Rightarrow p_{\alpha}(x, y)=0\right\}$;

(D2) $\forall_{\alpha \in \mathcal{A}} \forall_{x, y \in X}\left\{p_{\alpha}(x, y)=p_{\alpha}(y, x)\right\}$;

(D3) $\forall_{\alpha \in \mathcal{A}} \forall_{x, y, z \in X}\left\{p_{\alpha}(x, z) \preceq_{H} p_{\alpha}(x, y)+p_{\alpha}(y, z)\right\}$.

(ii) If $D$ is $D$-family, then the pair $(X, D)$ is called a cone uniform space.

(iii) A $P$-family $D$ is said to be separating if

(p4) $\forall_{x, y \in X}\left\{x \neq y \Rightarrow \exists_{\alpha \in \mathcal{A}}\left\{0 \prec_{H} p_{\alpha}(x, y)\right\}\right\}$.

(iv) If a $D$-family $D$ is separating, then the pair $(X, D)$ is called a Hausdorff cone uniform space.

A cone $H$ is said to be solid if $\operatorname{int}(H) \neq \emptyset$; $\operatorname{int}(H)$ denotes the interior of $H$. We will write $a \ll_{H} b$ to indicate that $b-a \in \operatorname{int}(H)$.

Definition 2.2. Let $L$ be an ordered normed space with solid cone $H$ and let $(X, D)$ be a cone uniform space with cone $H$.

(i) We say that a sequence $\left(w_{m}: m \in \mathbb{N}\right)$ in $X$ is a $p$-convergent in $X$, if there exists $w \in X$ such that $\forall_{\alpha \in \mathcal{A}} \forall_{c \in L, 0 \ll_{H} c} \exists_{n_{0} \in \mathbb{N}} \forall_{m \in \mathbb{N} ; n_{0} \leqslant m}\left\{p_{\alpha}\left(w_{m}, w\right) \ll_{H} c\right\}$.

(ii) We say that a sequence $\left(w_{m}: m \in \mathbb{N}\right)$ in $X$ is a $p$-Cauchy sequence in $X$, if $\forall_{\alpha \in \mathcal{A}} \forall_{c \in L, 0 \ll{ }_{H} c} \exists_{n_{0} \in \mathbb{N}} \forall_{m, n \in \mathbb{N} ; n_{0} \leqslant m<n}\left\{p_{\alpha}\left(w_{m}, w_{n}\right) \ll_{H} c\right\}$.

(iii) If every $p$-Cauchy sequence in $X$ is $p$-convergent in $X$, then $(X, p)$ is called a $p$ sequentially complete cone uniform space.

(iv) The set-valued dynamic system $(X, T)$ is called a cone closed set-valued dynamic system in $X$ if whenever $\left(w_{m}: m \in \mathbb{N}\right)$ is a sequence in $X p$-converging to $w \in X$ and $\left(v_{m}: m \in \mathbb{N}\right)$ is a sequence $p$-converging to $v \in X$ such that $v_{m} \in T\left(w_{m}\right)$ for all $m \in \mathbb{N}$, then $v \in T(w)$.

(v) Let $(X, D)$ be a $D$-sequentially complete cone uniform space. For an arbitrary subset $E$ of $X$, the cone closure of $E$, denoted by $\operatorname{cl}(E)$, is defined as the set $\operatorname{cl}(E)=\{w \in X$ : $\left.\exists_{\left(w_{m}: m \in \mathbb{N}\right) \subset E} \forall_{\alpha \in \mathscr{A}} \forall_{c \in L, 0<{ }_{H} c} \exists_{n_{0} \in \mathbb{N}} \forall_{m \in \mathbb{N} ; n_{0} \leqslant m}\left\{p_{\alpha}\left(w_{m}, w\right) \ll_{H} c\right\}\right\}$. The subset $E$ of $X$ is said to be a cone closed subset in $X$ if $\operatorname{cl}(E)=E$.

The cone $H$ is normal if a real number $M>0$ exists such that for each $a, b \in H$, $0 \preceq_{H} a \preceq_{H} b$ implies $\|a\| \leqslant M\|b\|$. The number $M$ satisfying the above is called the normal constant of $H$.

The following holds. 
Theorem 2.3 (see [22]). Let $L$ be an ordered normed space with normal solid cone $H$ and let $(X, D)$ be a cone uniform space with cone $H$.

(a) Let $\left(w_{m}: m \in \mathbb{N}\right)$ be a sequence in $X$ and let $w \in X$. Then the sequence $\left(w_{m}: m \in \mathbb{N}\right)$ is p-convergent to $w$ if and only if

$$
\forall_{\alpha \in \mathscr{A}} \forall_{\varepsilon>0} \exists_{n_{0} \in \mathbb{N}} \forall_{m \in \mathbb{N} ; n_{0} \leqslant m}\left\{\left\|p_{\alpha}\left(w_{m}, w\right)\right\|<\varepsilon\right\} .
$$

(b) Let $\left(w_{m}: m \in \mathbb{N}\right)$ be a sequence in $X$. Then the sequence $\left(w_{m}: m \in \mathbb{N}\right)$ is a $p$-Cauchy sequence if and only if

$$
\forall_{\alpha \in \mathcal{A}} \forall_{\varepsilon>0} \exists_{n_{0} \in \mathbb{N}} \forall_{m, n \in \mathbb{N} ; n_{0} \leqslant m<n}\left\{\left\|p_{\alpha}\left(w_{m}, w_{n}\right)\right\|<\varepsilon\right\} .
$$

(c) Each $\mathbf{p}$-convergent sequence is a $\mathbf{D}$-Cauchy sequence.

Definition 2.4. Let $L$ be an ordered normed space with normal solid cone $H$ and let $(X, p)$ be a cone uniform space with cone $H$.

(i) The family $\partial=\left\{J_{\alpha}: X \times X \rightarrow L, \alpha \in \mathcal{A}\right\}$ is said to be a 2 -family of cone pseudodistances on $X$ (2-family on $X$, for short) if the following three conditions hold:

(21) $\forall_{\alpha \in \mathscr{A}} \forall_{x, y \in X}\left\{0 \leq_{H} J_{\alpha}(x, y)\right\}$;

(22) $\forall_{\alpha \in \mathcal{A}} \forall_{x, y, z \in X}\left\{J_{\alpha}(x, z) \varliminf_{H} J_{\alpha}(x, y)+J_{\alpha}(y, z)\right\}$;

(23) for any sequence $\left(w_{m}: m \in \mathbb{N}\right)$ in $X$ such that

$$
\forall_{\alpha \in \mathcal{A}} \forall_{\varepsilon>0} \exists_{n_{0} \in \mathbb{N}} \forall_{m, n \in \mathbb{N}} ; n_{0} \leqslant m \leqslant n\left\{\left\|J_{\alpha}\left(w_{m}, w_{n}\right)\right\|<\varepsilon\right\},
$$

if there exists a sequence $\left(v_{m}: m \in \mathbb{N}\right)$ in $X$ satisfying

$$
\forall_{\alpha \in \mathscr{A}} \forall_{\varepsilon>0} \exists_{n_{0} \in \mathbb{N}} \forall_{m \in \mathbb{N}} ; n_{0} \leqslant m\left\{\left\|J_{\alpha}\left(w_{m}, v_{m}\right)\right\|<\varepsilon\right\},
$$

then

$$
\forall_{\alpha \in \mathscr{A}} \forall_{\varepsilon>0} \exists_{n_{0} \in \mathbb{N}} \forall_{m \in \mathbb{N} ; n_{0} \leqslant m}\left\{\left\|p_{\alpha}\left(w_{m}, v_{m}\right)\right\|<\varepsilon\right\} .
$$

(ii) Let the family $\mathcal{\partial}=\left\{J_{\alpha}: X \times X \rightarrow L, \alpha \in \mathcal{A}\right\}$ be a 2 -family on $X$. We say that a sequence $\left(w_{m}: m \in \mathbb{N}\right)$ in $X$ is a 2 -Cauchy sequence in $X$ if (2.3) holds.

For other families of cone pseudodistances in cone uniform spaces and various applications, see [22, 23]. The following is a consequence of Definition 2.4(i).

Proposition 2.5. Let $(X, D)$ be a Hausdorff cone uniform space with cone $H$. Let the family $\partial=\left\{J_{\alpha}\right.$ : $X \times X \rightarrow L, \alpha \in \mathcal{A}\}$ be a 2-family. If $\forall_{\alpha \in \mathcal{A}}\left\{J_{\alpha}(x, y)=0 \wedge J_{\alpha}(y, x)=0\right\}$, then $x=y$. 
Proof. Let $x, y \in X$ be such that $\forall_{\alpha \in \mathcal{A}}\left\{J_{\alpha}(x, y)=0 \wedge J_{\alpha}(y, x)=0\right\}$. By (22), $\forall_{\alpha \in \mathcal{A}}\left\{J_{\alpha}(x, x) \leq_{H} J_{\alpha}(x, y)+J_{\alpha}(y, x)\right\}$. By $(21)$, this gives $\forall_{\alpha \in \mathcal{A}}\left\{J_{\alpha}(x, x)=0\right\}$. Thus, we get $\forall_{\alpha \in \mathscr{A}} \forall_{\varepsilon>0} \exists_{n_{0} \in \mathbb{N}} \forall_{m, n \in \mathbb{N} ; n_{0} \leqslant m \leqslant n}\left\{\left\|J_{\alpha}\left(w_{m}, w_{n}\right)\right\|<\varepsilon\right\}$ and $\forall_{\alpha \in \mathscr{A}} \forall_{\varepsilon>0} \exists_{n_{0} \in \mathbb{N}} \forall_{m \in \mathbb{N} ; n_{0} \leqslant m}\left\{\left\|J_{\alpha}\left(w_{m}, v_{m}\right)\right\|<\right.$ $\varepsilon\}$ where $w_{m}=x, v_{m}=y, m \in \mathbb{N}$, and, by $(23), \forall_{\alpha \in \mathcal{A}} \forall_{\varepsilon>0} \exists_{n_{0} \in \mathbb{N}} \forall_{m \in \mathbb{N}} n_{0} \leqslant m\left\{\left\|p_{\alpha}\left(w_{m}, v_{m}\right)\right\|<\varepsilon\right\}$, that is, $\forall_{\alpha \in \mathcal{A}} \forall_{\varepsilon>0}\left\{\left\|p_{\alpha}(x, y)\right\|<\varepsilon\right\}$. Hence, $\forall_{\alpha \in \mathcal{A}}\left\{p_{\alpha}(x, y)=0\right\}$ which, according to $(\not D)$, implies that $x=y$.

Now we introduce the following three kinds of dissipative set-valued dynamic systems with generalized pseudodistances in cone uniform spaces (conditions (ii)-(iv) below).

Definition 2.6. Let $(X, P)$ be a Hausdorff cone uniform space and let $(X, T)$ be a set-valued dynamic system. Let $\mathcal{\partial}=\left\{J_{\alpha}: X \times X \rightarrow L, \alpha \in \mathcal{A}\right\}$ be a 2 -family on $X$ and let $\Omega=\left\{\omega_{\alpha}: X \rightarrow L, \alpha \in \mathcal{A}\right\}$ be a family of maps such that

$$
\forall_{\alpha \in \mathcal{A}} \forall_{x \in X}\left\{0 \preceq_{H} \omega_{\alpha}(x)\right\}
$$

(i) We say that a sequence $\left(w_{m}: m \in\{0\} \cup \mathbb{N}\right)$ in $X$ is $(2, \Omega)$-admissible if

$$
\forall_{\alpha \in \mathcal{A}} \forall_{m \in\{0\} \cup \mathbb{N}}\left\{J_{\alpha}\left(w_{m}, w_{m+1}\right) \varliminf_{H} w_{\alpha}\left(w_{m}\right)-\omega_{\alpha}\left(w_{m+1}\right)\right\}
$$

(ii) If the following conditions are satisfied:

(C1) $\emptyset \neq X_{0} \subset X$; and

(C2) $x \in X_{0}$ if and only if there exists a $(2, \Omega)$-admissible dynamic process $\left(w_{m}\right.$ : $m \in\{0\} \cup \mathbb{N})$ starting at $w_{0}=x$ of the system $(X, T)$,

then we say that $T$ is weak $\left(2, \Omega ; X_{0}\right)$-dissipative on $X$

(iii) We say that $T$ is $(2, \Omega)$-dissipative on $X$ if, for each $x \in X$, each dynamic process $\left(w_{m}: m \in\{0\} \cup \mathbb{N}\right)$ starting at $w_{0}=x$ of the system $(X, T)$ is $(2, \Omega)$-admissible.

(iv) We say that $T$ is strictly $(2, \Omega)$-dissipative on $X$ if, for each $x \in X$, each generalized sequence of iterations $\left(w_{m}: m \in\{0\} \cup \mathbb{N}\right)$ starting at $w_{0}=x$ of the system $(X, T)$ is $(2, \Omega)$-admissible.

If one of the conditions (ii)-(iv) holds, then we say that $(X, T)$ is a dissipative set-valued dynamic system with respect to $(2, \Omega)$ (dissipative set-valued dynamic system, for short).

Remark 2.7. It is worth noticing that if a sequence $\left(w_{m}: m \in\{0\} \cup \mathbb{N}\right)$ in $X$ is $(2, \Omega)$-admissible, then, for each $k \in \mathbb{N}$, a sequence $\left(w_{m+k}: m \in\{0\} \cup \mathbb{N}\right)$ is $(2, \Omega)$-admissible. Consequently, if $T$ is weak $\left(2, \Omega ; X_{0}\right)$-dissipative on $X, x \in X_{0}$, and $\left(w_{m}: m \in\{0\} \cup \mathbb{N}\right)$ is a dynamic process starting at $w_{0}=x$ of the system $(X, T)$ which is $(2, \Omega)$-admissible, then $\forall_{m \in \mathbb{N}}\left\{w_{m} \in X_{0}\right\}$; in general, $T\left(X_{0}\right) \neq X_{0}$ (see Example 7.3).

Now we can give the following conclusion. 
Proposition 2.8. Let $(X, D)$ be a Hausdorff cone uniform space and let $(X, T)$ be a set-valued dynamic system.

(a) If $T$ is weak $\left(2, \Omega ; X_{0}\right)$-dissipative on $X$, then $\left(X_{0}, \mathbb{K}_{2 ; T}\right)$ is a set-valued dynamic system where, for each $x \in X_{0}$,

$$
\begin{aligned}
\mathbb{K}_{2 ; T}(x)= & \bigcup\left\{\left\{w_{0}, w_{1}, w_{2}, \ldots\right\}:\left(w_{m}: m \in\{0\} \cup \mathbb{N}\right) \in \mathcal{K}_{2}(T, x)\right\}, \\
\mathcal{K}_{2}(T, x)= & \left\{\left(w_{m}: m \in\{0\} \cup \mathbb{N}\right),\right. \\
& \left.w_{0}=x: \forall_{m \in\{0\} \cup \mathbb{N}}\left\{w_{m+1} \in T\left(w_{m}\right) \wedge \forall_{\alpha \in \mathcal{A}}\left\{J_{\alpha}\left(w_{m}, w_{m+1}\right) \varliminf_{H} w_{\alpha}\left(w_{m}\right)-w_{\alpha}\left(w_{m+1}\right)\right\}\right\}\right\} .
\end{aligned}
$$

(b) If $T$ is $(2, \Omega)$-dissipative on $X$, then $\left(X, \mathbb{W}_{2 ; T}\right)$ is a set-valued dynamic system where, for each $x \in X$,

$$
\begin{gathered}
\mathbb{W}_{2 ; T}(x)=\bigcup\left\{\left\{w_{0}, w_{1}, w_{2}, \ldots\right\}:\left(w_{m}: m \in\{0\} \cup \mathbb{N}\right) \in \mathcal{W}_{2}(T, x)\right\}, \\
\mathcal{W}_{2}(T, x)=\left\{\left(w_{m}: m \in\{0\} \cup \mathbb{N}\right), w_{0}=x: \forall_{m \in\{0\} \cup \mathbb{N}}\left\{w_{m+1} \in T\left(w_{m}\right)\right\}\right\} .
\end{gathered}
$$

(c) If T is strictly $(2, \Omega)$-dissipative on $X$, then $\left(X, \mathbb{S}_{2 ; T}\right)$ is a set-valued dynamic system where, for each $x \in X$,

$$
\begin{array}{r}
\mathbb{S}_{2 ; T}(x)=\bigcup\left\{\left\{w_{0}, w_{1}, w_{2}, \ldots\right\}:\left(w_{m}: m \in\{0\} \cup \mathbb{N}\right) \in \mathcal{S}_{2}(T, x)\right\}, \\
\mathcal{S}_{2}(T, x)=\left\{\left(w_{m}: m \in\{0\} \cup \mathbb{N}\right), w_{0}=x: \forall_{m \in\{0\} \cup \mathbb{N}}\left\{w_{m+1} \in T^{[m+1]}\left(w_{0}\right)\right\}\right\} .
\end{array}
$$

Proof. The fact that

$$
\mathbb{K}_{2 ; T}: X_{0} \longrightarrow 2^{X_{0}}, \quad \mathbb{W}_{2 ; T}: X \longrightarrow 2^{X}, \quad \mathbb{S}_{2 ; T}: X \longrightarrow 2^{X}
$$

follows from (1.1), (1.2), Definition 2.6, Remark 2.7, and (2.8)-(2.13).

Remark 2.9. By Proposition 2.8 and Definition 2.6, we get:

(i) If $T$ is $(2, \Omega)$-dissipative on $X$, then $T$ is weak $\left(2, \Omega ; X_{0}\right)$-dissipative on $X$ for $X_{0}=X$ and $\forall_{x \in X_{0}}\left\{\mathbb{K}_{2 ; T}(x)=\mathbb{W}_{2 ; T}(x)\right\}$.

(ii) If $T$ is strictly $(2, \Omega)$-dissipative on $X$, then $T$ is $(2, \Omega)$-dissipative on $X$ and $\forall_{x \in X}\left\{\mathbb{W}_{2 ; T}(x) \subset \mathbb{S}_{2 ; T}(x)\right\}$.

Definition 2.10. Let $L$ be an ordered normed space with solid cone $H$. The cone $H$ is called regular if for every incresing (decresing) sequence which is bounded from above (below), that is, if for each sequence $\left(c_{m}: m \in \mathbb{N}\right)$ in $L$ such that $c_{1} \preceq_{H} c_{2} \preceq_{H} \cdots \preceq_{H} c_{m} \preceq_{H} \cdots \preceq_{H} b\left(b \preceq_{H} \cdots \preceq_{H} c_{m} \preceq_{H} \cdots \preceq_{H} c_{2} \preceq_{H} c_{1}\right)$ for some $b \in L$, there exists $c \in L$ such that $\lim _{m \rightarrow \infty}\left\|c_{m}-c\right\|=0$.

Remark 2.11. Every regular cone is normal; see [24]. 


\section{Periodic Point and Convergence Theorem for Weak $\left(2, \Omega ; X_{0}\right)$-Dissipative, $(2, \Omega)$-Dissipative, and Strictly $(2, \Omega)$-Dissipative Set-Valued Dynamic Systems in Cone Uniform Spaces}

Our main result of this section is the following.

Theorem 3.1. Let $L$ be an ordered Banach space with a regular solid cone $H$ and let $(X, D)$ be a Hausdorff sequentially complete cone uniform space with cone $H$. Let $\partial=\left\{J_{\alpha}: X \times X \rightarrow L, \alpha \in \mathbb{A}\right\}$ be a 2-family on $X$ and let $\Omega=\left\{\omega_{\alpha}: X \rightarrow L, \alpha \in A\right\}$ be a family of maps such that $\forall_{\alpha \in \mathcal{A}} \forall_{x \in X}\left\{0 \preceq_{H} \omega_{\alpha}(x)\right\}$. Let $(X, T)$ be a set-valued dynamic system. The following hold.

(a) If $T$ is weak $\left(2, \Omega ; X_{0}\right)$-dissipative on $X$, then, for each $x \in X_{0}$ and for each dynamic process $\left(w_{m}: m \in\{0\} \cup \mathbb{N}\right) \in \mathcal{K}_{2}(T, x)$, there exists $w \in \operatorname{cl}\left(X_{0}\right)$ such that $\left(w_{m}: m \in\{0\} \cup \mathbb{N}\right)$ is $D$-convergent to $w$. If, in addition, the map $T^{[q]}$ is cone closed in $X$ for some $q \in \mathbb{N}$, then $w \in T^{[q]}(w)$.

(b) If $T$ is $(2, \Omega)$-dissipative on $X$, then, for each $x \in X$ and for each dynamic process $\left(w_{m}\right.$ : $m \in\{0\} \cup \mathbb{N}) \in \mathcal{W}_{2}(T, x)$, there exists $w \in X$ such that $\left(w_{m}: m \in\{0\} \cup \mathbb{N}\right)$ is $p$ convergent to $w$. If, in addition, the map $T^{[q]}$ is cone closed in $X$ for some $q \in \mathbb{N}$, then $w \in T^{[q]}(w)$.

(c) If $T$ is strictly $(2, \Omega)$-dissipative on $X$, then, for each $x \in X$ and for each generalized sequence of iterations $\left(w_{m}: m \in\{0\} \cup \mathbb{N}\right) \in \mathcal{S}_{2}(T, x)$, there exists $w \in X$ such that $\left(w_{m}: m \in\{0\} \cup \mathbb{N}\right)$ is $p$-convergent to $w$. If, in addition, the map $T^{[q]}$ is cone closed in $X$ for some $q \in \mathbb{N}$, then, for each $x \in X$, there exists a generalized sequence of iterations $\left(w_{m}: m \in\{0\} \cup \mathbb{N}\right) \in \mathcal{S}_{2}(T, x)$ and $w \in X$ such that $\left(w_{m}: m \in\{0\} \cup \mathbb{N}\right)$ is $p$-convergent to $w$ and $w \in T^{[q]}(w)$.

Proof. The proof will be broken into three steps.

Step 1. Let (i) $x \in X_{0}$ and $\left(w_{m}: m \in\{0\} \cup \mathbb{N}\right) \in \mathcal{K}_{2}(T, x)$; or (ii) $x \in X$ and ( $w_{m}: m \in$ $\{0\} \cup \mathbb{N}) \in \mathcal{W}_{2}(T, x) \cup \mathcal{S}_{2}(T, x)$. We show that $\left(w_{m}: m \in\{0\} \cup \mathbb{N}\right)$ is 2 -Cauchy and $p$-Cauchy, that is,

$$
\begin{aligned}
& \forall_{\alpha \in \mathcal{A}} \forall_{\varepsilon>0} \exists_{n_{0} \in \mathbb{N}} \forall_{m, n \in \mathbb{N}} ; n_{0} \leqslant m \leqslant n \\
& \forall_{\alpha \in \mathscr{A}} \forall_{\varepsilon>0} \exists_{n_{0} \in \mathbb{N}} \forall_{m, n \in \mathbb{N} ; n_{0} \leqslant m<n}\left\{\left\|J_{\alpha}\left(w_{m}, w_{n}\right)\right\|<\varepsilon\right\}, \\
& \left.\left(w_{m}, w_{n}\right) \|<\varepsilon\right\},
\end{aligned}
$$

respectively; see Definitions 2.4(ii) and 2.2(ii) and Theorem 2.3(b).

Indeed, since $\leq_{L}$ is transitive, by (2.9), (2.11), (2.13), Definition 2.6(ii)-(iv) and (21), we get that $\forall_{\alpha \in \mathcal{A}} \forall_{m \in\{0\} \cup \mathbb{N}}\left\{\omega_{\alpha}\left(w_{m+1}\right) \leq_{H} \omega_{\alpha}\left(w_{m}\right)\right\}$. According to (2.6), for each $\alpha \in \mathcal{A}$, the sequence $\left(\omega_{\alpha}\left(w_{m}\right): m \in\{0\} \cup \mathbb{N}\right)$ is contained in $H$, bounded from below and, by the above, nonincreasing. Since $H$ is a closed and regular cone, it follows that

$$
\forall_{\alpha \in \mathcal{A}} \exists_{u_{\alpha} \in H}\left\{\lim _{m \rightarrow \infty}\left\|\omega_{\alpha}\left(w_{m}\right)-u_{\alpha}\right\|=0\right\} .
$$


Let now $\alpha_{0} \in \mathcal{A}$ and $\varepsilon_{0}>0$ be arbitrary and fixed. By (3.3),

$$
\exists_{n_{0} \in \mathbb{N}} \forall_{m ; n_{0} \leqslant m}\left\{\left\|\omega_{\alpha_{0}}\left(w_{m}\right)-u_{\alpha_{0}}\right\|<\frac{\varepsilon_{0}}{2 M}\right\}
$$

where $M$ is a normal constant of $H$ (see Remark 2.11). Furthermore, for $n_{0}<m \leqslant n$, using (21), (22), and (2.7), $0 \leq_{H} J_{\alpha_{0}}\left(w_{m}, w_{n}\right) \varliminf_{H} \sum_{k=m}^{n-1} J_{\alpha_{0}}\left(w_{k}, w_{k+1} \preceq_{H} w_{\alpha_{0}}\left(w_{m}\right)-\omega_{\alpha_{0}}\left(w_{n}\right)\right.$ and next, by (3.4) and the fact that $H$ is normal (see Remark 2.11), $\left\|J_{\alpha_{0}}\left(w_{m}, w_{n}\right)\right\| \leqslant M\left\|\omega_{\alpha_{0}}\left(w_{m}\right)-\omega_{\alpha_{0}}\left(w_{n}\right)\right\|$ $=M\left\|\omega_{\alpha_{0}}\left(w_{m}\right)-u_{\alpha_{0}}-\omega_{\alpha_{0}}\left(w_{n}\right)+u_{\alpha_{0}}\right\| \leqslant M\left\|\omega_{\alpha_{0}}\left(w_{m}\right)-u_{\alpha_{0}}\right\|+M\left\|\omega_{\alpha_{0}}\left(w_{n}\right)-u_{\alpha_{0}}\right\| \leqslant \varepsilon_{0} / 2+\varepsilon_{0} / 2=\varepsilon_{0}$. Therefore, (3.1) holds.

Also we can show that (3.2) holds. Indeed, by (3.1), $\quad \forall_{\alpha \in \mathcal{A}} \forall_{\varepsilon>0} \exists_{n_{0} \in \mathbb{N}} \forall_{m \geqslant n_{0}}$ $\forall_{k \in\{0\} \cup \mathbb{N}}\left\{\left\|J_{\alpha}\left(w_{m}, w_{k+m}\right)\right\|<\varepsilon\right\}$. Hence, if $i_{0} \in \mathbb{N}$ and $j_{0} \in\{0\} \cup \mathbb{N}$ where $i_{0}>j_{0}$ and

$$
u_{m}=w_{i_{0}+m}, \quad v_{m}=w_{j_{0}+m} \quad \text { for } m \in \mathbb{N},
$$

then we obtain

$$
\forall_{\alpha \in \mathscr{A}} \forall_{\varepsilon>0} \exists_{n_{0} \in \mathbb{N}} \forall_{m \geqslant n_{0}}\left\{\left\|J_{\alpha}\left(w_{m}, u_{m}\right)\right\|<\varepsilon \wedge\left\|J_{\alpha}\left(w_{m}, v_{m}\right)\right\|<\varepsilon\right\}
$$

We obtain according to (3.1), (3.6), and (23) that

$$
\forall_{\alpha \in \mathscr{A}} \forall_{\varepsilon>0} \exists_{n_{0} \in \mathbb{N}} \forall_{m \geqslant n_{0}}\left\{\left\|p_{\alpha}\left(w_{m}, u_{m}\right)\right\|<\varepsilon \wedge\left\|p_{\alpha}\left(w_{m}, v_{m}\right)\right\|<\varepsilon\right\} .
$$

By (3.5), from (3.7) it follows that

$$
\forall_{\alpha \in \mathcal{A}} \forall_{\varepsilon>0} \exists_{n_{0} \in \mathbb{N}} \forall_{m \geqslant n_{0}}\left\{\left\|p_{\alpha}\left(w_{m}, w_{i_{0}+m}\right)\right\|<\frac{\varepsilon}{2 M} \wedge\left\|p_{\alpha}\left(w_{m}, w_{j_{0}+m}\right)\right\|<\frac{\varepsilon}{2 M}\right\} .
$$

Next, if $n_{0} \leqslant m<n$, then $n=i_{0}+n_{0}$ and $m=j_{0}+n_{0}$ for some $i_{0} \in \mathbb{N}$ and $j_{0} \in\{0\} \cup \mathbb{N}$ such that $i_{0}>j_{0}$. Thus, by $(D 1)-(D 3), \forall_{\alpha \in \mathcal{A}}\left\{0 \leq_{H} p_{\alpha}\left(w_{m}, w_{n}\right)=p_{\alpha}\left(w_{i_{0}+n_{0}}, w_{j_{0}+n_{0}}\right) \leq_{H} p_{\alpha}\left(w_{n_{0}}, w_{i_{0}+n_{0}}\right)+\right.$ $\left.p_{\alpha}\left(w_{n_{0}}, w_{j_{0}+n_{0}}\right)\right\}$. Using (3.8), this gives $\forall_{\alpha \in \mathcal{A}}\left\{\left\|p_{\alpha}\left(w_{m}, w_{n}\right)\right\| \leqslant M\left\|p_{\alpha}\left(w_{n_{0}}, w_{i_{0}+n_{0}}\right)\right\|+\right.$ $\left.M\left\|p_{\alpha}\left(w_{n_{0}}, w_{j_{0}+n_{0}}\right)\right\|<\varepsilon / 2+\varepsilon / 2=\varepsilon\right\}$. The proof of (3.2) is complete.

Step 2. Assertions (a) and (b) hold.

Indeed, let (i) $x \in X_{0}$ and $\left(w_{m}: m \in\{0\} \cup \mathbb{N}\right) \in \mathcal{K}_{2}(T, x)$; or (ii) $x \in X$ and $\left(w_{m}: m \in\right.$ $\{0\} \cup \mathbb{N}) \in \mathcal{W}_{2}(T, x)$.

Since $\forall_{m \in\{0\} \cup \mathbb{N}}\left\{w_{m} \in \mathbb{K}_{2 ; T}(x)\right\}$ or $\forall_{m \in\{0\} \cup \mathbb{N}}\left\{w_{m} \in \mathbb{W}_{2 ; T}(x)\right\}, X$ is a Hausdorff and sequentially complete cone space and (2.14) holds, therefore, by virtue of Step 1, Proposition 2.8 and Theorem 2.3(b) and (c), we claim that $\left(w_{m}: m \in\{0\} \cup \mathbb{N}\right)$ is a $p$-Cauchy sequence and there exists a unique $w \in \operatorname{cl}\left(\mathbb{K}_{2 ; T}(x)\right)$ or $w \in \mathrm{cl}\left(\mathbb{W}_{2 ; T}(x)\right)$, respectively, where $\mathbb{K}_{2 ; T}(x) \subset X_{0}, \mathbb{W}_{2 ; T}(x) \subset X$, and $\operatorname{cl}(X)=X$, such that the sequence $\left(w_{m}: m \in\{0\} \cup \mathbb{N}\right)$ is $p$-convergent to $w$, that is, $\forall_{\alpha \in \mathcal{A}}\left\{\lim _{m \rightarrow \infty}\left\|p_{\alpha}\left(w_{m}, w\right)\right\|=0\right\}$.

Now we see that if $T^{[q]}$ is cone closed for some $q \in \mathbb{N}$, then the point $w$ satisfies $w \in T^{[q]}(w)$. Indeed, by (2.9) or (2.11), we conclude that

$$
\forall_{m \in \mathbb{N}}\left\{w_{m} \in T\left(w_{m-1}\right) \subset T^{[2]}\left(w_{m-2}\right) \subset \cdots \subset T^{[m-1]}\left(w_{1}\right) \subset T^{[m]}\left(w_{0}\right)\right\},
$$


which gives

$$
w_{m q+k} \in T^{[q]}\left(w_{(m-1) q+k}\right) \quad \text { for } k=1,2, \ldots, q, m \in \mathbb{N}
$$

Since $T^{[q]}$ is cone closed in $X$ and $\lim _{m \rightarrow \infty} w_{m}=w$, therefore, by (3.10) and Definition 2.2(iv), we get $w \in T^{[q]}(w)$.

Step 3. Assertion (c) holds.

Indeed, if $x \in X$ and $\left(w_{m}: m \in\{0\} \cup \mathbb{N}\right) \in \mathcal{S}_{2}(T, x)$, then, by virtue of Step 1, Proposition 2.8 and Theorem 2.3(b) and (c), we claim that $\forall_{m \in\{0\} \cup \mathbb{N}}\left\{w_{m} \in T^{[m]}(x) \subset \mathbb{S}_{2 ; T}(x) \subset\right.$ $X\},\left(w_{m}: m \in\{0\} \cup \mathbb{N}\right)$ is a $p$-Cauchy sequence, and there exists a unique $w \in \operatorname{cl}\left(\mathbb{S}_{2 ; T}(x)\right)$ such that the sequence $\left(w_{m}: m \in\{0\} \cup \mathbb{N}\right) p$-converges to $w$.

If $T^{[q]}$ is cone closed in $X$ for some $q \in \mathbb{N}$, then, for each $x \in X$, by Remark 2.9(b) and Step 2 (part (b)), there exists a generalized sequence of iterations $\left(w_{m}: m \in\{0\} \cup \mathbb{N}\right) \in$ $\mathcal{S}_{2}(T, x) \cap \mathcal{W}_{2}(T, x) \subset \mathcal{S}_{2}(T, x)$ satisfying (3.9) and (3.10) and there exists $w \in \operatorname{cl}\left(\mathbb{W}_{2 ; T}(x)\right) \subset$ $\operatorname{cl}\left(\mathbb{S}_{2 ; T}(x)\right) \subset X$ such that $\left(w_{m}: m \in\{0\} \cup \mathbb{N}\right)$ is $p$-convergent to $w$ and $w \in T^{[q]}(w)$.

\section{Dissipative Set-Valued Dynamic Systems with Generalized Pseudodistances in Uniform Spaces}

Let $(X, \Phi)$ be a Hausdorff uniform space with uniformity defined by a saturated family $\Phi=$ $\left\{d_{\alpha}: \alpha \in \mathcal{A}\right\}$ of pseudometrics $d_{\alpha}, \alpha \in \mathcal{A}$, uniformly continuous on $X^{2}$.

Definition 4.1. Let $(X, \Phi)$ be a Hausdorff uniform space. The family $u=\left\{U_{\alpha}: X \times X \rightarrow[0, \infty)\right.$, $\alpha \in \mathcal{A}\}$ is said to be a $\mathcal{U}$-family of generalized pseudodistances on $X$ (U-family, for short) if the following two conditions hold:

(U1) $\forall_{\alpha \in \mathcal{A}} \forall_{x, y, z \in X}\left\{U_{\alpha}(x, z) \leqslant U_{\alpha}(x, y)+U_{\alpha}(y, z)\right\}$;

$(\mathfrak{U} 2)$ for any sequence $\left(w_{m}: m \in \mathbb{N}\right)$ in $X$ such that

$$
\forall_{\alpha \in \mathcal{A}}\left\{\lim _{m \rightarrow \infty} \sup _{n>m} U_{\alpha}\left(w_{m}, w_{n}\right)=0\right\}
$$

if there exists a sequence $\left(v_{m}: m \in \mathbb{N}\right)$ in $X$ satisfying

$$
\forall_{\alpha \in \mathcal{A}}\left\{\lim _{m \rightarrow \infty} U_{\alpha}\left(w_{m}, v_{m}\right)=0\right\}
$$

then

$$
\forall_{\alpha \in \mathcal{A}}\left\{\lim _{m \rightarrow \infty} d_{\alpha}\left(w_{m}, v_{m}\right)=0\right\}
$$

Definition 4.2. Let $(X, \Phi)$ be a Hausdorff uniform space and let $(X, T)$ be a set-valued dynamic system. Let the family $\mathcal{U}=\left\{U_{\alpha}: X \times X \rightarrow[0, \infty), \alpha \in \mathcal{A}\right\}$ be a $\mathcal{U}$-family and let $\Gamma=\left\{\gamma_{\alpha}: X \rightarrow\right.$ $[0,+\infty), \alpha \in \mathcal{A}\}$ be a family of maps. 
Fixed Point Theory and Applications

(i) We say that a sequence $\left(w_{m}: m \in\{0\} \cup \mathbb{N}\right)$ in $X$ is $(\mathcal{U}, \Gamma)$-admissible if

$$
\forall_{\alpha \in \mathcal{A}} \forall_{m \in\{0\} \cup \mathbb{N}}\left\{U_{\alpha}\left(w_{m}, w_{m+1}\right) \leqslant \gamma_{\alpha}\left(w_{m}\right)-\gamma_{\alpha}\left(w_{m+1}\right)\right\}
$$

(ii) If the following conditions are satisfied:

(C1) $\emptyset \neq X_{0} \subset X$;

(C2) $x \in X_{0}$ if and only if there exists a $(\mathcal{U}, \Gamma)$-admissible dynamic process $\left(w_{m}\right.$ : $m \in\{0\} \cup \mathbb{N})$ starting at $w_{0}=x$ of the system $(X, T)$,

then we say that $T$ is weak $\left(\mathcal{U}, \Gamma ; X_{0}\right)$-dissipative on $X$.

(iii) We say that $T$ is $(\mathcal{U}, \Gamma)$-dissipative on $X$ if, for each $x \in X$, each dynamic process $\left(w_{m}: m \in\{0\} \cup \mathbb{N}\right)$ starting at $w_{0}=x$ of the system $(X, T)$ is $(\mathcal{U}, \Gamma)$-admissible.

(iv) We say that $T$ is strictly $(\mathcal{U}, \Gamma)$-dissipative on $X$ if, for each $x \in X$, each generalized sequence of iterations $\left(w_{m}: m \in\{0\} \cup \mathbb{N}\right)$ starting at $w_{0}=x$ of the system $(X, T)$ is $(\mathfrak{U}, \Gamma)$-admissible.

If one of the conditions (ii)-(iv) holds, then we say that $(X, T)$ is a dissipative set-valued dynamic system with respect to $(\mathcal{U}, \Gamma)$ (dissipative set-valued dynamic system, for short).

Proposition 4.3. Let $(X, \Phi)$ be a Hausdorff uniform space and let $(X, T)$ be a set-valued dynamic system.

(a) If $T$ is weak $\left(\mathcal{U}, \Gamma ; X_{0}\right)$-dissipative on $X$, then $\left(X_{0}, \mathbb{K}_{\mathfrak{U} ; T}\right)$ is a set-valued dynamic system where, for each $x \in X_{0}, \mathbb{K}_{\mathfrak{u} ; T}(x)=\bigcup\left\{\left\{w_{0}, w_{1}, w_{2}, \ldots\right\}:\left(w_{m}: m \in\{0\} \cup \mathbb{N}\right) \in\right.$ $\left.\mathcal{K}_{\mathfrak{U}}(T, x)\right\}$ and $\mathcal{K}_{\mathfrak{U}}(T, x)=\left\{\left(w_{m}: m \in\{0\} \cup \mathbb{N}\right), w_{0}=x: \forall_{m \in\{0\} \cup \mathbb{N}}\left\{w_{m+1} \in\right.\right.$ $\left.\left.T\left(w_{m}\right) \wedge \forall_{\alpha \in \mathcal{A}}\left\{U_{\alpha}\left(w_{m}, w_{m+1}\right) \leqslant \gamma_{\alpha}\left(w_{m}\right)-\gamma_{\alpha}\left(w_{m+1}\right)\right\}\right\}\right\}$.

(b) If $T$ is $(\mathcal{U}, \Gamma)$-dissipative on $X$, then $\left(X, \mathbb{W}_{\mathcal{U} ; T}\right)$ is a set-valued dynamic system where, for each $x \in X, \mathbb{W}_{\mathcal{U} ; T}(x)=\bigcup\left\{\left\{w_{0}, w_{1}, w_{2}, \ldots\right\}:\left(w_{m}: m \in\{0\} \cup \mathbb{N}\right) \in \mathcal{W}_{\mathcal{U}}(T, x)\right\}$, and $\mathcal{W}_{\mathfrak{U}}(T, x)=\left\{\left(w_{m}: m \in\{0\} \cup \mathbb{N}\right), w_{0}=x: \forall_{m \in\{0\} \cup \mathbb{N}}\left\{w_{m+1} \in T\left(w_{m}\right)\right\}\right\}$.

(c) If $T$ is strictly $(\mathcal{U}, \Gamma)$-dissipative on $X$, then $\left(X, \mathbb{S}_{\mathcal{U} ; T}\right)$ is a set-valued dynamic system where, for each $x \in X, \mathbb{S}_{\mathcal{U} ; T}(x)=\bigcup\left\{\left\{w_{0}, w_{1}, w_{2}, \ldots\right\}:\left(w_{m}: m \in\{0\} \cup \mathbb{N}\right) \in \mathcal{S}_{\mathcal{U}}(T, x)\right\}$ and $\mathcal{S}_{\mathfrak{U}}(T, x)=\left\{\left(w_{m}: m \in\{0\} \cup \mathbb{N}\right), w_{0}=x: \forall_{m \in\{0\} \cup \mathbb{N}}\left\{w_{m+1} \in T^{[m+1]}\left(w_{0}\right)\right\}\right\}$.

\section{Periodic Point and Convergence Theorem for Weak $\left(\mathcal{U}, \Gamma ; X_{0}\right)$-Dissipative, $(\mathcal{U}, \Gamma)$-Dissipative, and Strictly $(\mathcal{U}, \Gamma)$-Dissipative Set-Valued Dynamic Systems in Uniform Spaces}

Let $\left(\Lambda, \geq_{\Lambda}\right)$ denote a directed set whose elements will be indicated by the letters $\lambda, \eta, \mu$. Let $T: X \rightarrow 2^{Y}$ where $X$ and $Y$ are topological spaces.

The following are equivalent: (a) the map $T$ is closed, that is, the graph of $T$ is closed in $X \times X ;(b)$ whenever $\left(w_{\lambda}: \lambda \in \Lambda\right)$ is a net converging to $w$ and $\left(v_{\lambda}: \lambda \in \Lambda\right)$ is a net converging to $v$ such that $v_{\lambda} \in T\left(w_{\lambda}\right)$ for all $\lambda \in \Lambda$, then $v \in T(w)$. Recall that the graph of $T$ is $\{(x, y): x \in X, y \in T(x)\}$.

The map $T$ is called upper semicontinuous at $w \in X$ if for each open set $G$ containing $T(w)$ there exists a neighbourhood $N(w)$ of $w$ such that $T(v) \subset G$ for each $v \in N(w)$ and 
upper semicontinuous in $X$ if it is upper semicontinuous at each point $w$ of $X$ and $T(w)$ is compact for each $w \in X$; see Berge [25, page 111].

Remark 5.1. It is known that

(i) if the map $T$ is closed, then, for each $x \in X$, the set $T(x)$ is closed [25, page 111] but, generally, the converse is not possible [26, Example 7.19, page 75].

(ii) every upper semicontinuous map is closed [25, Theorem 6, page 112] and, if $X$ is a compact space, then the map is closed if and only if it is upper semicontinuous [25, Corollary, page 112].

Definition 5.2. Let $(X, \Phi)$ be a Hausdorff uniform space.

(i) We say that a sequence $\left(w_{m}: m \in \mathbb{N}\right)$ in $X$ is a Cauchy sequence in $X$ if $\forall_{\alpha \in \mathcal{A}}\left\{\lim _{m \rightarrow \infty} \sup _{n>m} d_{\alpha}\left(w_{m}, w_{n}\right)=0\right\}$.

(ii) We say that a sequence $\left(w_{m}: m \in \mathbb{N}\right)$ in $X$ converges in $X$, if there exists $w \in X$ such that $\forall_{\alpha \in \mathcal{A}}\left\{\lim _{m \rightarrow \infty} d_{\alpha}\left(w_{m}, w\right)=0\right\}$.

(iii) If every Cauchy sequence in $X$ is convergent in $X$, then $(X, \Phi)$ is called a sequentially complete uniform space.

(iv) Let the family $\mathcal{U}=\left\{U_{\alpha}: X \times X \rightarrow[0, \infty), \alpha \in \mathcal{A}\right\}$ be a $\mathcal{U}$-family on $X$. We say that a sequence $\left(w_{m}: m \in \mathbb{N}\right)$ in $X$ is a $\mathcal{U}$-Cauchy sequence in $X$ if $\forall_{\alpha \in \mathcal{A}}\left\{\lim _{m \rightarrow \infty} \sup _{n>m} U_{\alpha}\left(w_{m}, w_{n}\right)=0\right\}$.

Consequence of Theorem 3.1 is the following.

Theorem 5.3. Let $(X, \Phi)$ be a Hausdorff sequentially complete uniform space and let $(X, T)$ be a setvalued dynamic system. Let the family $\mathcal{u}=\left\{U_{\alpha}: X \times X \rightarrow[0, \infty), \alpha \in \mathcal{A}\right\}$ be a $\mathcal{U}$-family and let $\left.\Gamma=\left\{\gamma_{\alpha}: X \rightarrow 0,+\infty\right], \alpha \in \mathcal{A}\right\}$ be a family of proper maps. The following hold.

(a) If $T$ is weak $\left(\mathcal{U}, \Gamma ; X_{0}\right)$-dissipative on $X$, then, for each $x \in X_{0}$ and for each dynamic process $\left(w_{m}: m \in\{0\} \cup \mathbb{N}\right) \in \mathcal{K}_{\mathfrak{U}}(T, x)$, there exists $w \in \operatorname{cl}\left(X_{0}\right)$ such that $\left(w_{m}: m \in\{0\} \cup \mathbb{N}\right)$ is convergent to $w$. If, in addition, the map $T^{[q]}$ is closed (or $T^{[q]}$ is upper semicontinuous) in $X$ for some $q \in \mathbb{N}$, then $w \in T^{[q]}(w)$.

(b) If $T$ is $(\mathcal{U}, \Gamma)$-dissipative on $X$, then, for each $x \in X$ and for each dynamic process $\left(w_{m}\right.$ : $m \in\{0\} \cup \mathbb{N}) \in \mathcal{W}_{\mathfrak{U}}(T, x)$, there exists $w \in X$ such that $\left(w_{m}: m \in\{0\} \cup \mathbb{N}\right)$ is convergent to $w$. If, in addition, the map $T^{[q]}$ is closed (or $T^{[q]}$ is upper semicontinuous) in $X$ for some $q \in \mathbb{N}$, then $w \in T^{[q]}(w)$.

(c) If $T$ is strictly $(\mathcal{U}, \Gamma)$-dissipative on $X$, then, for each $x \in X$ and for each generalized sequence of iterations $\left(w_{m}: m \in\{0\} \cup \mathbb{N}\right) \in \mathcal{S}_{\mathfrak{U}}(T, x)$, there exists $w \in X$ such that $\left(w_{m}: m \in\{0\} \cup \mathbb{N}\right)$ is convergent to $w$. If, in addition, the map $T^{[q]}$ is closed (or $T^{[q]}$ is upper semicontinuous ) in $X$ for some $q \in \mathbb{N}$, then, for each $x \in X$, there exists a generalized sequence of iterations $\left(w_{m}: m \in\{0\} \cup \mathbb{N}\right) \in \mathcal{S}_{\mathcal{U}}(T, x)$ and $w \in X$ such that $\left(w_{m}: m \in\{0\} \cup \mathbb{N}\right)$ is convergent to $w$ and $w \in T^{[q]}(w)$. 
Fixed Point Theory and Applications

\section{Endpoint and Convergence Theorem for $(\mathcal{U}, \Gamma)$-Dissipative and Strictly $(\mathcal{U}, \Gamma)$-Dissipative Set-Valued Dynamic Systems in Uniform Spaces}

We recall the following.

Definition 6.1. Let $(X, \tau)$ be a topological space and let $(X, T)$ be a set-valued dynamic system. The map $T$ is called lower semicontinuous at $w \in X$ (written: lsc at $w \in X$ ) if and only if for any $v \in T(w)$ and for any net $\left(w_{\lambda}: \lambda \in \Lambda\right)$ of elements $w_{\lambda} \in X, \lambda \in \Lambda, \tau$-converging to $w$, there exists a net $\left(v_{\lambda}: \lambda \in \Lambda\right)$ of elements $v_{\lambda} \in T\left(w_{\lambda}\right), \lambda \in \Lambda, \tau$-converging to $v$. The map $T$ is called lsc on $X$ if it is lsc at every point of $w \in X$.

The main result of this section is what follows.

Theorem 6.2. Let $(X, \Phi)$ be a Hausdorff sequentially complete uniform space and let $(X, T)$ be a setvalued dynamic system. Let the family $\mathcal{U}=\left\{U_{\alpha}: X \times X \rightarrow[0, \infty), \alpha \in \mathcal{A}\right\}$ be a $U$-family and let $\left.\Gamma=\left\{\gamma_{\alpha}: X \rightarrow 0, \infty\right), \alpha \in \mathcal{A}\right\}$ be a family of maps.

Assume that $T$ is $(\mathcal{U}, \Gamma)$-dissipative on $X$. Then the following hold.

$\left(\mathrm{a}_{1}\right)$ The map $\mathbb{W}_{\mathfrak{U}_{T} T}$ is $(\mathcal{U}, \Gamma)$-dissipative on $X$ and, for each $x \in X$, there exist ( $w_{m}: m \in$ $\{0\} \cup \mathbb{N}) \in \mathcal{W}_{\mathcal{U}}\left(\mathbb{W}_{\mathcal{U}_{T},}, x\right)$ and $w \in X$ such that $\left(w_{m}: m \in\{0\} \cup \mathbb{N}\right)$ converges to $w$ and $\bigcap_{m \geqslant 0} \operatorname{cl}\left(\mathbb{W}_{\mathcal{U}_{;},}\left(w_{m}\right)\right)=\{w\}$.

$\left(\mathrm{a}_{2}\right)$ If, for each $x \in X, \mathbb{W}_{\mathcal{U}_{T} T}(x)$ is a closed set, then $\operatorname{End}(T) \neq \emptyset$ and, for each $x \in X$, there exist $\left(w_{m}: m \in\{0\} \cup \mathbb{N}\right) \in \mathcal{W}_{\mathcal{U}}\left(\mathbb{W}_{\mathcal{U}_{T}, T}, x\right)$ and $w \in \operatorname{End}(T)$ such that $\left(w_{m}: m \in\{0\} \cup \mathbb{N}\right)$ converges to $w$ and $\bigcap_{m \geqslant 0} \mathbb{W}_{\mathcal{u} ; T}\left(w_{m}\right)=\{w\}$.

(a) If $T$ is lsc on $X$, then $\operatorname{End}(T) \neq \emptyset$ and, for each $x \in X$, there exist $\left(w_{m}: m \in\{0\} \cup \mathbb{N}\right) \in$ $\mathcal{W}_{\mathcal{U}}\left(\mathbb{W}_{\mathcal{U}_{i},}, x\right)$ and $w \in \operatorname{End}(T)$ such that $\left(w_{m}: m \in\{0\} \cup \mathbb{N}\right)$ converges to $w$ and $\bigcap_{m \geqslant 0} \operatorname{cl}\left(\mathbb{W}_{\mathcal{U}_{i T}}\left(w_{m}\right)\right)=\{w\}$.

Assume that $T$ is strictly $(\mathcal{U}, \Gamma)$-dissipative on $X$. Then the following hold.

$\left(\mathrm{b}_{1}\right)$ The map $\mathbb{S}_{\mathcal{U} ; T}$ is strictly $(\mathcal{U}, \Gamma)$-dissipative on $X$ and, for each $x \in X$, there exist ( $w_{m}: m \in$ $\{0\} \cup \mathbb{N}) \in \mathcal{S}_{\mathcal{U}}\left(\mathbb{S}_{\mathcal{U} ; T}, x\right)$ and $w \in X$ such that $\left(w_{m}: m \in\{0\} \cup \mathbb{N}\right)$ converges to $w$ and $\bigcap_{m \geqslant 0} \operatorname{cl}\left(\mathbb{S}_{\mathcal{U} ; T}\left(w_{m}\right)\right)=\{w\}$.

$\left(b_{2}\right)$ If, for each $x \in X, \mathbb{S}_{\mathcal{U} ; T}(x)$ is a closed set, then $\operatorname{End}(T) \neq \emptyset$ and, for each $x \in X$, there exist $\left(w_{m}: m \in\{0\} \cup \mathbb{N}\right) \in \mathcal{S}_{\mathcal{U}}\left(\mathbb{S}_{\mathcal{U} ; T}, x\right)$ and $w \in \operatorname{End}(T)$ such that $\left(w_{m}: m \in\{0\} \cup \mathbb{N}\right)$ converges to $w$ and $\bigcap_{m \geqslant 0} \mathbb{S}_{\mathcal{U} ; T}\left(w_{m}\right)=\{w\}$.

$\left(b_{3}\right)$ If $T$ is lsc on $X$, then $\operatorname{End}(T) \neq \emptyset$ and, for each $x \in X$, there exist ( $w_{m}: m \in\{0\} \cup$ $\mathbb{N}) \in \mathcal{S}_{\mathcal{U}}\left(\mathbb{S}_{\mathcal{U} ; T}, x\right)$ and $w \in \operatorname{End}(T)$ such that $\left(w_{m}: m \in\{0\} \cup \mathbb{N}\right)$ converges to $w$ and $\bigcap_{m \geqslant 0} \operatorname{cl}\left(\mathbb{S}_{\mathcal{u} ; T}\left(w_{m}\right)\right)=\{w\}$.

Proof. $\left(\mathrm{a}_{1}\right)$ The proof of $\left(\mathrm{a}_{1}\right)$ will be broken into six steps.

Step 1. We show that $\forall_{\alpha \in \mathcal{A}} \forall_{x \in X} \forall_{y \in \mathbb{W}_{\mathcal{M} ; T}(x)}\left\{U_{\alpha}(x, y) \leqslant \gamma_{\alpha}(x)-\gamma_{\alpha}(y)\right\}$.

Indeed, let $x \in X$ and $y \in \mathbb{W}_{\mathcal{U} ; T}(x)$ be arbitrary and fixed. By definition of $\mathbb{W}_{\mathcal{U} ; T}(x)$, there exist a dynamic process $\left(w_{m}: m \in\{0\} \cup \mathbb{N}\right) \in \mathcal{W}_{\mathfrak{U}}(T, x)$ starting at $w_{0}=x$ of the system $(X, T)$ and $m_{0} \in\{0\} \cup \mathbb{N}$ such that $y=w_{m_{0}}$; recall that then (1.1) and (4.4) hold (i.e., $\forall_{m \in\{0\} \cup \mathbb{N}}\left\{w_{m+1} \in T\left(w_{m}\right)\right\}$ and $\forall_{\alpha \in \mathcal{A}} \forall_{m \in\{0\} \cup \mathbb{N}}\left\{U_{\alpha}\left(w_{m}, w_{m+1}\right) \leqslant \gamma_{\alpha}\left(w_{m}\right)-\gamma_{\alpha}\left(w_{m+1}\right)\right\}$ 
hold). By virtue of $(\mathcal{U} 1)$, this gives $\forall_{\alpha \in \mathcal{A}}\left\{U_{\alpha}(x, y) \leqslant \sum_{m=0}^{m_{0}-1} U_{\alpha}\left(w_{m}, w_{m+1}\right) \leqslant \sum_{m=0}^{m_{0}-1}\left[\gamma_{\alpha}\left(w_{m}\right)-\right.\right.$ $\left.\left.\gamma_{\alpha}\left(w_{m+1}\right)\right]=\gamma_{\alpha}(x)-\gamma_{\alpha}(y)\right\}$.

Step 2. We show that $\mathbb{W}_{\mathcal{U}_{T} T}$ is $(\mathcal{U}, \Gamma)$-dissipative on $X$.

If $x \in X$ and $\left(w_{m}: m \in\{0\} \cup \mathbb{N}\right)$ is a dynamic process starting at $w_{0}$ $=x$ of the system $\left(X, \mathbb{W}_{\mathfrak{U} ; T}\right)$, that is, $\forall_{m \in\{0\} \cup \mathbb{N}}\left\{w_{m+1} \in \mathbb{W}_{\mathfrak{U} ; T}\left(w_{m}\right)\right\}$, then, by Step 1, $\forall_{\alpha \in \mathscr{A}} \forall_{m \in\{0\} \cup \mathbb{N}}\left\{U_{\alpha}\left(w_{m}, w_{m+1}\right) \leqslant \gamma_{\alpha}\left(w_{m}\right)-\gamma_{\alpha}\left(w_{m+1}\right)\right\}$, that is, $\left(w_{m}: m \in\{0\} \cup \mathbb{N}\right)$ is $(\mathcal{U}, \Gamma)-$ admissible. This gives that $\mathbb{W}_{\mathcal{U} ; T}$ is $(\mathcal{U}, \Gamma)$-dissipative on $X$.

Step 3. We show that $\forall_{x \in X}\left\{\mathcal{W}_{\mathfrak{U}}(T, x) \subset \mathcal{W}_{\mathfrak{U}}\left(\mathbb{W}_{\mathfrak{U} ; T}, x\right)\right\}$.

Indeed, by Proposition 4.3(b), for $x \in X, \mathcal{W}_{\mathfrak{U}}(T, x)=\left\{\left(w_{m}: m \in\{0\} \cup \mathbb{N}\right)\right.$ : $\left.\forall_{m \in\{0\} \cup \mathbb{N}}\left\{w_{m+1} \in T\left(w_{m}\right)\right\}, w_{0}=x\right\}$. Next, by Step $2, \mathbb{W}_{\mathcal{U} ; T}$ is $(\mathcal{U}, \Gamma)$-dissipative on $X$. Consequently,

$$
\forall_{x \in X}\left\{\mathcal{W}_{\mathcal{U}}\left(\mathbb{W}_{\mathcal{u} ; T}, x\right)=\left\{\left(c_{m}: m \in\{0\} \cup \mathbb{N}\right): \forall_{m \in\{0\} \cup \mathbb{N}}\left\{c_{m+1} \in \mathbb{W}_{\mathcal{U} ; T}\left(c_{m}\right)\right\}, c_{0}=x\right\}\right\},
$$

where

$$
\begin{aligned}
\forall_{m \in\{0\} \cup \mathbb{N}}\left\{\mathbb{W}_{\mathcal{U} ; T}\left(c_{m}\right)\right. & =\bigcup\left\{\left\{s_{0}, s_{1}, s_{2}, \ldots\right\}:\left(s_{j}: j \in\{0\} \cup \mathbb{N}\right) \in \mathcal{W}_{\mathcal{U}}\left(T, c_{m}\right)\right\} \\
& \left.=\bigcup\left\{\left\{s_{0}, s_{1}, s_{2}, \ldots\right\}: s_{0}=c_{m}, s_{j} \in T\left(s_{j-1}\right), j \in \mathbb{N}\right\}\right\} .
\end{aligned}
$$

Let now $\left(w_{m}: m \in\{0\} \cup \mathbb{N}\right) \in \mathcal{W}_{\mathcal{U}}(T, x)$. Then $\forall_{m \in\{0\} \cup \mathbb{N}}\left\{w_{m+1} \in T\left(w_{m}\right)\right\}$. Hence $\forall_{m \in\{0\} \cup \mathbb{N}} \forall_{k \geqslant m}\left\{w_{k+1} \in T\left(w_{k}\right)\right\}$. Thus $\forall_{m \in\{0\} \cup \mathbb{N}}\left\{\left(w_{m}, w_{m+1}, w_{m+2}, \ldots\right) \in w_{\mathfrak{u}}\left(T, w_{m}\right)\right\}$. Hence, by (6.2), $\forall_{m \in\{0\} \cup \mathbb{N}}\left\{\left\{w_{m}, w_{m+1}, w_{m+2}, \ldots\right\} \subset \mathbb{W}_{\mathcal{U} ; T}\left(w_{m}\right)\right\}$. In particular, $\forall_{m \in\{0\} \cup \mathbb{N}}\left\{w_{m+1} \in\right.$ $\left.\mathbb{W}_{\mathfrak{u} ; T}\left(w_{m}\right)\right\}$. By $(6.1),\left(w_{m}: m \in\{0\} \cup \mathbb{N}\right) \in \mathcal{W}_{\mathfrak{u}}\left(\mathbb{W}_{\mathfrak{u}_{T},}, x\right)$.

Step 4. If $x \in X$ and $\left(w_{m}: m \in\{0\} \cup \mathbb{N}\right) \in \mathcal{W}_{\mathcal{U}}\left(\mathbb{W}_{\mathcal{u} ; T}, x\right)$, then

$$
\forall_{\alpha \in \mathscr{A}} \forall_{m \in\{0\} \cup \mathbb{N}}\left\{\gamma_{\alpha}\left(w_{m+1}\right) \leqslant \gamma_{\alpha}\left(w_{m}\right)\right\}
$$

Indeed, by (6.1), $\forall_{m \in\{0\} \cup \mathbb{N}}\left\{w_{m+1} \in \mathbb{W}_{\mathfrak{U} ; T}\left(w_{m}\right)\right\}, w_{0}=x$. Hence, by virtue of the Step 2 and Definition 4.2(iii), the sequence $\left(w_{m}: m \in\{0\} \cup \mathbb{N}\right)$ is $(\mathcal{U}, \Gamma)$-admissible, that is, $\forall_{\alpha \in \mathcal{A}} \forall_{m \in\{0\} \cup \mathbb{N}}\left\{U_{\alpha}\left(w_{m}, w_{m+1}\right) \leqslant \gamma_{\alpha}\left(w_{m}\right)-\gamma_{\alpha}\left(w_{m+1}\right)\right\}$. By definitions of $\mathcal{U}$ and $\Gamma$, this gives (6.3).

Step 5. Let

$$
\forall_{\alpha \in \mathscr{A}} \forall_{x \in X}\left\{\Delta_{\alpha}\left(\mathbb{W}_{\mathcal{U} ; T}(x)\right)=\sup \left\{U_{\alpha}(x, t): t \in \mathbb{W}_{\mathcal{U} ; T}(x)\right\}\right\}
$$

and let

$$
\forall_{\alpha \in \mathcal{A}} \forall_{x \in X}\left\{\rho_{\alpha ; \mathbb{W}_{u ; T}}(x)=\inf \left\{\gamma_{\alpha}(t): t \in \mathbb{W}_{\mathcal{U} ; T}(x)\right\}\right\} .
$$


Then

$$
\forall_{\alpha \in \mathcal{A}} \forall_{x \in X}\left\{\Delta_{\alpha}\left(\mathbb{W}_{\mathcal{U} ; T}(x)\right) \leqslant \gamma_{\alpha}(x)-\rho_{\alpha ; \mathbb{W}}(x)\right\}
$$

Indeed, if $\alpha \in \mathcal{A}$ and $x \in X$, then, by Step 1 , we get $\Delta_{\alpha}\left(\mathbb{W}_{\mathcal{u}_{;} T}(x)\right)=\sup \left\{U_{\alpha}(x, t): t \in\right.$ $\left.\mathbb{W}_{u_{i T}}(x)\right\} \leqslant \sup \left\{\gamma_{\alpha}(x)-\gamma_{\alpha}(t): t \in \mathbb{W}_{u_{i T}}(x)\right\} \leqslant \gamma_{\alpha}(x)-\rho_{\alpha ; \mathbb{W}_{\mathcal{U}} T}(x)$.

Step 6. Let $\delta_{\alpha}(E)=\sup \left\{d_{\alpha}\left(y_{1}, y_{2}\right): y_{1}, y_{2} \in E\right\}, E \in 2^{X}, \alpha \in \mathcal{A}$. Then, for each $x \in X$, there exist a dynamic process $\left(w_{m}: m \in\{0\} \cup \mathbb{N}\right) \in \mathcal{W}_{\mathfrak{U}}\left(\mathbb{W}_{\mathfrak{U} ; T}, x\right)$ and a unique $w \in X$ such that

$$
\begin{gathered}
\forall_{\alpha \in \mathcal{A}}\left\{\lim _{m \rightarrow \infty} \sup _{n>m} U_{\alpha}\left(w_{m}, w_{n}\right)=0\right\}, \\
\forall_{\alpha \in \mathcal{A}}\left\{\lim _{m \rightarrow \infty} \sup _{n>m} d_{\alpha}\left(w_{m}, w_{n}\right)=0\right\}, \\
\forall_{\alpha \in \mathcal{A}}\left\{\lim _{m \rightarrow \infty} d_{\alpha}\left(w_{m}, w\right)=0\right\}, \\
\forall_{\alpha \in \mathcal{A}}\left\{\lim _{m \rightarrow \infty} \delta_{\alpha}\left(\mathbb{W}_{\mathcal{u} ; T}\left(w_{m}\right)\right)=\lim _{m \rightarrow \infty} \delta_{\alpha}\left(\operatorname{cl}\left(\mathbb{W}_{\mathcal{U} ; T}\left(w_{m}\right)\right)\right)=0\right\}, \\
\bigcap_{m \geqslant 0} \operatorname{cl}\left(\mathbb{W}_{\mathcal{U} ; T}\left(w_{m}\right)\right)=\{w\} .
\end{gathered}
$$

Indeed, first, let us observe that since $T$ is $(\mathcal{U}, \Gamma)$-dissipative on $X$ and $\forall_{x \in X}\{x \in$ $\left.\mathbb{W}_{\mathcal{U} ; T}(x)\right\}$ (i.e., $\forall_{x \in X}\left\{\mathbb{W}_{\mathcal{U} ; T}(x) \neq \emptyset\right\}$ ), thus there exists

$$
\forall_{\alpha \in \mathscr{A}} \forall_{x \in X}\left\{\rho_{\alpha ; \mathbb{W}}(x)=\inf \left\{\gamma_{\alpha}(t): t \in \mathbb{W}_{\mathcal{U} ; T}(x)\right\}\right\} .
$$

Now let $x \in X$ and $\alpha_{0} \in \mathcal{A}$ be arbitrary and fixed. Defining $w_{0}=x$, since $T$ is $(\mathcal{U}, \Gamma)$-dissipative on $X$ and $w_{0} \in \mathbb{W}_{\mathcal{U} ; T}\left(w_{0}\right)$, by (6.2), we have that

$$
\mathbb{W}_{\mathcal{U} ; T}\left(w_{0}\right)=\bigcup\left\{\left\{s_{0}, s_{1}, s_{2}, \ldots\right\}: s_{0}=w_{0}, s_{j} \in T\left(s_{j-1}\right), j \in \mathbb{N}\right\} \neq \emptyset
$$

Therefore, by (6.12) and (6.13), there exists $w_{1} \in \mathbb{W}_{\mathcal{U} ; T}\left(w_{0}\right)$ such that $\gamma_{\alpha_{0}}\left(w_{1}\right) \leqslant \rho_{\alpha_{0} ; \mathbb{W}_{\mathcal{U}} ;}\left(w_{0}\right)+1$. Similarly, there exists $w_{2} \in \mathbb{W}_{\mathcal{U}_{i} T}\left(w_{1}\right)$ such that $\gamma_{\alpha_{0}}\left(w_{2}\right) \leqslant \rho_{\alpha_{0} ; \mathbb{W}_{\mathcal{u}_{i}}}\left(w_{1}\right)+2^{-1}$. By induction, we may construct a dynamic process $\left(w_{m}: m \in\{0\} \cup \mathbb{N}\right) \in \mathcal{W}_{\mathfrak{U}}\left(\mathbb{W}_{\mathfrak{U} ; T}, x\right)$ satisfying (see (6.1), (6.2), and (6.3) and Step 1)

$$
\begin{gathered}
\forall_{m \in \mathbb{N}}\left\{w_{m} \in \mathbb{W}_{\mathcal{u} ; T}\left(w_{m-1}\right)\right\} \\
\forall_{m \in\{0\} \cup \mathbb{N}}\left\{\gamma_{\alpha_{0}}\left(w_{m+1}\right) \leqslant \rho_{\alpha_{0} ; \mathbb{W} u_{i T}}\left(w_{m}\right)+2^{-m}\right\},
\end{gathered}
$$

where $\forall_{m \in \mathbb{N}}\left\{\varrho_{\alpha_{0} ; \mathbb{W}_{\mathcal{u}_{i}}}\left(w_{m}\right)=\inf \left\{\gamma_{\alpha_{0}}(t): t \in \mathbb{W}_{\mathcal{u} ; T}\left(w_{m}\right)\right\}\right\}$. Next, by (6.2),

$$
\forall_{m \in \mathbb{N}}\left\{\mathbb{W}_{\mathfrak{U}_{; T}}\left(w_{m}\right) \subset \mathbb{W}_{\mathfrak{u}_{i T}}\left(w_{m-1}\right)\right\}
$$


which gives

$$
\forall_{m \in\{0\} \cup \mathbb{N}}\left\{\varrho_{\alpha_{0} ; \mathbb{W} \mathcal{W}_{T}}\left(w_{m}\right) \leqslant \varrho_{\alpha_{0} ; \mathbb{W} \mathcal{W}_{T}}\left(w_{m+1}\right)\right\} .
$$

By (6.6), (6.15), and (6.17), $\Delta_{\alpha_{0}}\left(\mathbb{W}_{\mathcal{u} ; T}\left(w_{m+1}\right)\right) \leqslant \gamma_{\alpha_{0}}\left(w_{m+1}\right)-\rho_{\alpha_{0} ; \mathbb{W}_{u ; T}}\left(w_{m+1}\right) \leqslant \rho_{\alpha_{0} ; \mathbb{W} u ; T}\left(w_{m}\right)+$ $\left.2^{-m}-\rho_{\alpha_{0} ; \mathbb{W}} ; w_{m}\left(w_{m}\right)\right] \leqslant 2^{-m}$ which implies

$$
\lim _{m \rightarrow \infty} \Delta_{\alpha_{0}}\left(\mathbb{W}_{\mathfrak{u} ; T}\left(w_{m+1}\right)\right)=0
$$

Moreover, by (6.14) and (6.16), for arbitrary and fixed $m \in \mathbb{N}$, we have that

$$
\forall_{n>m}\left\{\left\{w_{m}, w_{n}\right\} \subset \mathbb{W}_{\mathfrak{U} ; T}\left(w_{m}\right)\right\}
$$

Next, by (6.19), we have

$$
\forall_{n>m}\left\{U_{\alpha_{0}}\left(w_{m}, w_{n}\right) \leqslant \sup \left\{U_{\alpha_{0}}\left(w_{m}, t\right): t \in \mathbb{W}_{\mathcal{U} ; T}\left(w_{m}\right)\right\}=\Delta_{\alpha_{0}}\left(\mathbb{W}_{\mathcal{U}_{; T}}\left(w_{m}\right)\right\}\right.
$$

Therefore, (6.20) and (6.18) imply (6.7).

Using (6.7) and analogous argument as in Step 1 of the proof of Theorem 3.1, we obtain (6.8). Indeed, from (6.7), $\forall_{\alpha \in \mathscr{A}} \forall_{\varepsilon>0} \exists_{n_{1} \in \mathbb{N}} \forall_{m>n_{1}}\left\{\sup \left\{U_{\alpha}\left(w_{m}, w_{n}\right): n>m\right\}<\varepsilon\right\}$ and, in particular, $\forall_{\alpha \in \mathcal{A}} \forall_{\varepsilon>0} \exists_{n_{1} \in \mathbb{N}} \forall_{m>n_{1}} \forall_{k \in \mathbb{N}}\left\{U_{\alpha}\left(w_{m}, w_{k+m}\right)<\varepsilon\right\}$. If $i_{0}, j_{0} \in \mathbb{N}, i_{0}>j_{0}$, are arbitrary and fixed and $u_{m}=w_{i_{0}+m}$ and $v_{m}=w_{j_{0}+m}, m \in \mathbb{N}$, this gives $\forall_{\alpha \in \mathcal{A}}\left\{\lim _{m \rightarrow \infty} U_{\alpha}\left(w_{m}, u_{m}\right)=\right.$ $\left.\lim _{m \rightarrow \infty} U_{\alpha}\left(w_{m}, v_{m}\right)=0\right\}$. By (6.7) and $(\mathcal{U} 2), \forall_{\alpha \in \mathcal{A}}\left\{\lim _{m \rightarrow \infty} d_{\alpha}\left(w_{m}, u_{m}\right)=\lim _{m \rightarrow \infty} d_{\alpha}\left(w_{m}, v_{m}\right)\right.$ $=0\}$. Hence

$$
\begin{aligned}
& \forall_{\alpha \in \mathscr{A}} \forall_{\varepsilon>0} \exists_{n_{2} \in \mathbb{N}} \forall_{m>n_{2}}\left\{d_{\alpha}\left(w_{m}, w_{i_{0}+m}\right)<\frac{\varepsilon}{2}\right\}, \\
& \forall_{\alpha \in \mathscr{A}} \forall_{\varepsilon>0} \exists_{n_{3} \in \mathbb{N}} \forall_{m>n_{3}}\left\{d_{\alpha}\left(w_{m}, w_{j_{0}+m}\right)<\frac{\varepsilon}{2}\right\} .
\end{aligned}
$$

Therefore, if $\alpha_{0} \in \mathcal{A}$ and $\varepsilon_{0}>0$ are arbitrary and fixed, $n_{0}=\max \left\{n_{2}, n_{3}\right\}+1$ and $k$, $l \in \mathbb{N}$ be arbitrary and fixed and such that $k>l>n_{0}$, then $k=i_{0}+n_{0}$ and $l=j_{0}+n_{0}$ for some $i_{0}, j_{0} \in \mathbb{N}$ such that $i_{0}>j_{0}$ and we get $d_{\alpha_{0}}\left(w_{k}, w_{l}\right)=d_{\alpha_{0}}\left(w_{i_{0}+n_{0}}, w_{j_{0}+n_{0}}\right) \leqslant d_{\alpha_{0}}\left(w_{n_{0}}, w_{i_{0}+n_{0}}\right)+$ $d_{\alpha_{0}}\left(w_{n_{0}}, w_{j_{0}+n_{0}}\right)<\varepsilon_{0} / 2+\varepsilon_{0} / 2=\varepsilon_{0}$.

Consequently, $\forall_{\alpha \in \mathscr{A}} \forall_{\varepsilon>0} \exists_{n \in \mathbb{N}} \forall_{k, l \in \mathbb{N}, k>l>n}\left\{d_{\alpha}\left(w_{k}, w_{l}\right)<\varepsilon\right\}$. The proof of (6.8) is complete.

By (6.8), there exists a unique $w \in X$ such that (6.9) holds.

Now we prove (6.10). With the aim of this, let $x_{m}, y_{m} \in \mathbb{W}_{\mathcal{U}_{; T}}\left(w_{m}\right), m \in \mathbb{N}$, be arbitrary and fixed. Then, by (6.18) and definition of $\Delta_{\alpha}\left(\mathbb{W}_{\mathcal{U} ; T}\left(w_{m}\right)\right)$, we have $\forall_{\alpha \in \mathcal{A}}\left\{\lim _{m \rightarrow \infty} U_{\alpha}\left(w_{m}\right.\right.$, $\left.\left.x_{m}\right)=\lim _{m \rightarrow \infty} U_{\alpha}\left(w_{m}, y_{m}\right)=0\right\}$. Hence, by (6.7) and $(\mathcal{U} 2), \forall_{\alpha \in \mathcal{A}}\left\{\lim _{m \rightarrow \infty} d_{\alpha}\left(w_{m}, x_{m}\right)=\right.$ $\left.\lim _{m \rightarrow \infty} d_{\alpha}\left(w_{m}, y_{m}\right)=0\right\}$ which gives $\forall_{\alpha \in \mathcal{A}}\left\{\lim _{m \rightarrow \infty} d_{\alpha}\left(x_{m}, y_{m}\right)=0\right\}$, that is, formula (6.10) holds.

Finally, let us observe that $X$ is sequentially complete and Hausdorff, inclusions (6.16) imply that the sequence of sets $\left\{\mathbb{W}_{\mathfrak{U}_{T} T}\left(w_{m}\right)\right\}$ has the property of finite intersections and that the properties (6.9), (6.10), and (6.14) hold. Consequently, (6.11) holds. 
$\left(a_{2}\right)$ From Proposition 4.3(b), Step 2 of the proof of $\left(a_{1}\right)$, we conclude that

$$
\forall_{x \in \mathrm{X}}\left\{T\left(\mathbb{W}_{\mathcal{U} ; T}(x)\right) \subset \mathbb{W}_{\mathcal{U} ; T}(x)\right\}
$$

and if the sequence $\left(w_{m}: m \in\{0\} \cup \mathbb{N}\right)$ is such as in Step 6 of the proof of $\left(\mathrm{a}_{1}\right)$, then, since, for each $x \in X$, the set $\mathbb{W}_{\mathcal{U}_{T} T}(x)$ is closed, using (6.11) and (6.22), we conclude that $T(w)$

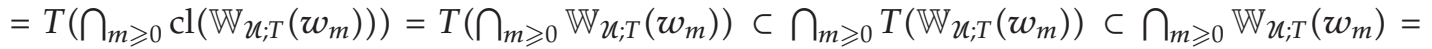
$\bigcap_{m \geqslant 0} \mathrm{cl}\left(\mathbb{W}_{\mathcal{u} ; T}\left(w_{m}\right)\right)=\{w\}$, that is, that $w$ is an endpoint of $T$.

$\left(\mathrm{a}_{3}\right)$ Let $x \in X$ be arbitrary and fixed and let the sequence $\left(w_{m}: m \in\{0\} \cup \mathbb{N}\right) \in$ $\mathcal{W}_{\mathcal{U}}\left(\mathbb{W}_{\mathcal{U} ; T}, x\right)$ be such as in Step 6 of the proof of $\left(\mathrm{a}_{1}\right)$. Then

$$
\forall_{m \in \mathbb{N}}\left\{T\left(\operatorname{cl}\left(\mathbb{W}_{\mathcal{U}_{i T}}\left(w_{m}\right)\right)\right) \subset \operatorname{cl}\left(\mathbb{W}_{\mathcal{U} ; T}\left(w_{m}\right)\right)\right\}
$$

Indeed, let $m_{0} \in \mathbb{N}$ be arbitrary and fixed. We prove that, if $u \in \mathrm{cl}\left(\mathbb{W}_{u ; T}\left(w_{m_{0}}\right)\right)$ and if $y \in T(u)$ is arbitrary and fixed, then $y \in \operatorname{cl}\left(\mathbb{W}_{u_{;} T}\left(w_{m_{0}}\right)\right)$. With the aim of this, we consider two cases.

Case 1. Assume that $u \in \mathbb{W}_{\mathfrak{u} ; T}\left(w_{m_{0}}\right)$. Then, by (6.22), we have that $y \in T(u) \subset \mathbb{W}_{\mathfrak{u} ; T}\left(w_{m_{0}}\right) \subset$ $\operatorname{cl}\left(\mathbb{W}_{\mathcal{U} ; T}\left(w_{m_{0}}\right)\right)$.

Case 2. Assume that $u \in \mathrm{cl}\left(\mathbb{W}_{\mathcal{U}_{; T}}\left(w_{m_{0}}\right)\right) \backslash \mathbb{W}_{\mathcal{u}_{i T}}\left(w_{m_{0}}\right)$ and let $\left(u_{\lambda}: \lambda \in \Lambda\right)$ be a net of elements $u_{\lambda} \in \mathbb{W}_{u_{;} T}\left(w_{m_{0}}\right), \lambda \in \Lambda$, which is convergent to $u$. Since $T$ is lsc at $u$ and $y \in T(u)$, by Definition 6.1 and the fact that $u \in \mathrm{cl}\left(\mathbb{W}_{u_{i} T}\left(w_{m_{0}}\right)\right) \subset X$ (cf. Proposition 4.3(b)), then there exists a net $\left(y_{\lambda}: \lambda \in \Lambda\right)$ of elements $y_{\lambda} \in T\left(u_{\lambda}\right), \lambda \in \Lambda$, which is convergent to $y$. However, since $u_{\curlywedge} \in \mathbb{W}_{\mathcal{u}_{i T}}\left(w_{m_{0}}\right), \lambda \in \Lambda$, thus, by (6.22), we have $T\left(u_{\lambda}\right) \subset \mathbb{W}_{u_{i} T}\left(w_{m_{0}}\right), \lambda \in \Lambda$, and, consequently, $y_{\lambda} \in T\left(u_{\lambda}\right) \subset \mathbb{W}_{u_{;} T}\left(w_{m_{0}}\right), \lambda \in \Lambda$. Since $\left(y_{\lambda}: \lambda \in \Lambda\right)$ is convergent to $y$, this gives that $y \in \operatorname{cl}\left(\mathbb{W}_{\mathcal{U} ; T}\left(w_{m_{0}}\right)\right)$.

Now, using $\left(\mathrm{a}_{1}\right)$ and (6.23), we get $T(w)=T\left(\bigcap_{m \geqslant 0} \mathrm{cl}\left(\mathbb{W}_{\mathcal{U}_{T} T}\left(w_{m}\right)\right)\right) \subset \bigcap_{m \geqslant 0}$ $T\left(\operatorname{cl}\left(\mathbb{W}_{\mathfrak{u}_{T} T}\left(w_{m}\right)\right)\right) \subset \bigcap_{m \geqslant 0} \mathrm{cl}\left(\mathbb{W}_{\mathfrak{u}_{T} T}\left(w_{m}\right)\right)=\{w\}$, that is, $w$ is an endpoint of $T$.

$\left(b_{1}\right)$ The proof of $\left(b_{1}\right)$ will be broken into sixt steps.

Step 7. The map $\mathbb{S}_{\mathcal{U} ; T}$ is strictly $(\mathcal{U}, \Gamma)$-dissipative on $X$.

Indeed, since

$$
\forall_{x \in X}\left\{\mathbb{S}_{\mathcal{U} ; T}(x)=\bigcup_{m \in\{0\} \cup \mathbb{N}} T^{[m]}(x)\right\}
$$

therefore

$$
\forall_{m \in \mathbb{N}} \forall_{x \in X}\left\{\left(\mathbb{S}_{\mathcal{U} ; T}\right)^{[m]}(x)=\mathbb{S}_{\mathcal{U} ; T}(x)\right\}
$$

On the other hand, by assumption that $T$ is strictly $(\mathcal{U}, \Gamma)$-dissipative on $X$, we have that if $x \in X$ is arbitrary and fixed and a generalized sequence of iterations $\left(w_{m}: m \in\{0\} \cup \mathbb{N}\right)$ is such that $w_{0}=x$ and $\forall_{m \in\{0\} \cup \mathbb{N}}\left\{w_{m+1} \in T^{[m+1]}(x)\right\}$, then $\forall_{\alpha \in \mathcal{A}} \forall_{m \in\{0\} \cup \mathbb{N}}\left\{U_{\alpha}\left(w_{m}, w_{m+1}\right) \leqslant\right.$ $\left.\gamma_{\alpha}\left(w_{m}\right)-\gamma_{\alpha}\left(w_{m+1}\right)\right\}$. However, then, by Proposition 4.3(c), $\left(w_{m}: m \in\{0\} \cup \mathbb{N}\right) \in \mathcal{S}_{\mathcal{U}}(T, x)$ and 
$\forall_{m \in\{0\} \cup \mathbb{N}}\left\{w_{m} \in \mathbb{S}_{\mathcal{U} ; T}(x)\right\}$. Using $(6.25)$, this gives $\forall_{m \in\{0\} \cup \mathbb{N}}\left\{w_{m} \in\left(\mathbb{S}_{\mathcal{U} ; T}\right)^{[m]}(x)\right\}$; remember that the sequence $\left(w_{m}: m \in\{0\} \cup \mathbb{N}\right)$ satisfies $\forall_{\alpha \in \mathcal{A}} \forall_{m \in\{0\} \cup \mathbb{N}}\left\{U_{\alpha}\left(w_{m}, w_{m+1}\right) \leqslant \gamma_{\alpha}\left(w_{m}\right)-\gamma_{\alpha}\left(w_{m+1}\right)\right\}$. By virtue of Proposition 4.3(c), and Definition 4.2(iv), this implies that $\mathbb{S}_{\mathcal{U} ; T}$ is strictly $(\mathcal{U}, \Gamma)$ dissipative on $X$.

Step 8. We show that $\forall_{x \in X}\left\{\mathcal{S}_{\mathcal{U}}(T, x) \subset \mathcal{S}_{\mathcal{U}}\left(\mathbb{S}_{\mathcal{U} ; T}, x\right)\right\}$.

Indeed, if $\left(w_{m}: m \in\{0\} \cup \mathbb{N}\right) \in \mathcal{S}_{\mathcal{U}}(T, x)$, then $\forall_{m \in\{0\} \cup \mathbb{N}}\left\{w_{m+1} \in T^{[m+1]}(x)\right\}$ which, by (6.24), implies that $\forall_{m \in\{0\} \cup \mathbb{N}}\left\{w_{m+1} \in_{k \in\{0\} \cup \mathbb{N}} T^{[k]}(x)=\mathbb{S}_{\mathcal{U} ; T}(x)\right\}, w_{0}=x$. Next, by Step 7, Definition 4.2(iv), Proposition 4.3(c) and (6.25), $\forall_{x \in X}\left\{\mathcal{S}_{\mathcal{U}}\left(\mathbb{S}_{\mathcal{U} ; T}, x\right)=\left\{\left(w_{m}: m \in\{0\} \cup \mathbb{N}\right)\right.\right.$ : $\left.\forall_{m \in\{0\} \cup \mathbb{N}}\left\{w_{m} \in\left(\mathbb{S}_{\mathcal{U} ; T}\right)^{[m]}(x)\right\}=\left\{\left(w_{m}: m \in\{0\} \cup \mathbb{N}\right): \forall_{m \in\{0\} \cup \mathbb{N}}\left\{w_{m} \in \mathbb{S}_{\mathcal{U} ; T}(x)\right\}\right\}\right\}$ where $w_{0}=x$ and $\left(\mathbb{S}_{\mathcal{U} ; T}\right)^{[0]}=I_{X}$. Consequently, $\left(w_{m}: m \in\{0\} \cup \mathbb{N}\right) \in \mathcal{S}_{\mathcal{U}}\left(\mathbb{S}_{\mathcal{U} ; T}, x\right)$.

Step 9. Let $x \in X$. If $\left(w_{m}: m \in\{0\} \cup \mathbb{N}\right) \in \mathcal{S}_{\mathcal{U}}\left(\mathbb{S}_{\mathcal{U} ; T}, x\right)$, then $\forall_{\alpha \in \mathscr{A}} \forall_{m \in\{0\} \cup \mathbb{N}}\left\{\gamma_{\alpha}\left(w_{m+1}\right) \leqslant \gamma_{\alpha}\left(w_{m}\right)\right\}$.

By (6.25) and Proposition 4.3(c), $\forall_{m \in\{0\} \cup \mathbb{N}}\left\{w_{m+1} \in \mathbb{S}_{\mathcal{U} \cdot T}^{[m+1]}(x)=\mathbb{S}_{\mathcal{U}_{i T}}(x)\right\}, w_{0}=x$, and then, by Step 7 and Definition 4.2(iv), $\forall_{\alpha \in \mathcal{A}} \forall_{m \in\{0\} \cup \mathbb{N}}\left\{U_{\alpha}\left(w_{m}, w_{m+1}\right) \leqslant \gamma_{\alpha}\left(w_{m}\right)-\gamma_{\alpha}\left(w_{m+1}\right)\right\}$.

Step 10. We have $\forall_{\alpha \in \mathscr{A}} \forall_{x \in X} \forall_{y \in \mathbb{S}_{\mathcal{M} ; T}(x)}\left\{U_{\alpha}(x, y) \leqslant \gamma_{\alpha}(x)-\gamma_{\alpha}(y)\right\}$.

Indeed, if $x \in X$ and $y \in \mathbb{S}_{\mathcal{U}_{i} T}(x)$, then there exist $m_{0} \in\{0\} \cup \mathbb{N}, y \in T^{\left[m_{0}+1\right]}(x)$ and $\left(w_{m}: m \in\{0\} \cup \mathbb{N}\right) \in \mathcal{S}_{\mathcal{U}}(T, x)$ such that $y=w_{m_{0}+1}$ and $w_{0}=x$. However, $\forall_{m \in\{0\} \cup \mathbb{N}}\left\{w_{m+1} \in\right.$ $\left.T^{[m+1]}(x)\right\}$ and $\forall_{\alpha \in \mathscr{A}} \forall_{m \in\{0\} \cup \mathbb{N}}\left\{U_{\alpha}\left(w_{m}, w_{m+1}\right) \leqslant \gamma_{\alpha}\left(w_{m}\right)-\gamma_{\alpha}\left(w_{m+1}\right)\right\}$. Hence, $\forall_{\alpha \in \mathcal{A}}\left\{U_{\alpha}(x, y) \leqslant\right.$ $\left.\sum_{m=0}^{m_{0}} U_{\alpha}\left(w_{m}, w_{m+1}\right) \leqslant \sum_{m=0}^{m_{0}}\left[\gamma_{\alpha}\left(w_{m}\right)-\gamma_{\alpha}\left(w_{m+1}\right)\right]=\gamma_{\alpha}(x)-\gamma_{\alpha}(y)\right\}$.

Step 11. We have $\forall_{\alpha \in \mathscr{A}} \forall_{x \in X}\left\{\Delta_{\alpha}\left(\mathbb{S}_{\mathcal{U} ; T}(x)\right) \leqslant \gamma_{\alpha}(x)-\varrho_{\alpha ; \mathbb{S}_{\mathcal{M} ; T}}(x)\right\}$ where $\forall_{\alpha \in \mathscr{A}} \forall_{x \in X}\left\{\Delta_{\alpha}\left(\mathbb{S}_{\mathcal{U} ; T}(x)\right)=\right.$ $\sup \left\{U_{\alpha}(x, t): t \in \mathbb{S}_{\mathcal{U} ; T}(x)\right\}$ and $\forall_{\alpha \in \mathscr{A}} \forall_{x \in X}\left\{\varrho_{\alpha ; \mathcal{S U}_{i T}}(x)=\inf \left\{\gamma_{\alpha}(t): t \in \mathbb{S}_{\mathcal{U} ; T}(x)\right\}\right\}$.

This is a consequence of the Step 10, (U⿱⺌兀), and $(\mathfrak{U} 2)$.

Step 12. For each $x \in X$, there exist a generalized sequence of iterations $\left(w_{m}: m \in\{0\} \cup \mathbb{N}\right) \in$ $\mathcal{S}_{\mathcal{U}}\left(\mathbb{S}_{\mathcal{U} ; T}, x\right)$ and a unique $w \in X$ such that

$$
\begin{aligned}
& \forall_{\alpha \in \mathcal{A}}\left\{\lim _{m \rightarrow \infty} \sup _{n>m} U_{\alpha}\left(w_{n}, w_{m}\right)=0\right\}, \\
& \forall_{\alpha \in \mathcal{A}}\left\{\lim _{m \rightarrow \infty} \sup _{n>m} d_{\alpha}\left(w_{n}, w_{m}\right)=0\right\}, \\
& \exists_{w \in X}\left\{\lim _{m \rightarrow \infty} w_{m}=w\right\}, \\
& \forall_{\alpha \in \mathcal{A}}\left\{\lim _{m \rightarrow \infty} \delta_{\alpha}\left(\mathbb{S}_{\mathcal{U} ; T}\left(w_{m}\right)\right)=\lim _{m \rightarrow \infty} \delta_{\alpha}\left(\operatorname{cl}\left(\mathbb{S}_{\mathcal{U} ; T}\left(w_{m}\right)\right)\right)=0\right\}, \\
& \bigcap_{m \geqslant 0} \operatorname{cl}\left(\mathbb{S}_{\mathcal{U} ; T}\left(w_{m}\right)\right)=\{w\} .
\end{aligned}
$$

This can be obtained by an analogous argument as in Step 6, using Steps 7-11.

$\left(b_{2}\right)$ We show that wis an endpoint of $T$. 
Indeed, $\forall_{x \in X}\left\{T\left(\mathbb{S}_{\mathcal{U}_{; T}}(x)\right) \subset \mathbb{S}_{\mathcal{U} ; T}(x)\right\}$ and assuming that $\left(w_{m}: m \in\{0\} \cup \mathbb{N}\right)$ and $w$ is such as in Step 12, then we conclude that the following holds $T(w)=T\left(\bigcap_{m \geqslant 0} \operatorname{cl}\left(\mathbb{S}_{\mathcal{U} ; T}\left(w_{m}\right)\right)\right)=$ $T\left(\bigcap_{m \geqslant 0} \mathbb{S}_{\mathcal{U} ; T}\left(w_{m}\right)\right) \subset \bigcap_{m \geqslant 0} T\left(\mathbb{S}_{\mathcal{U} ; T}\left(w_{m}\right)\right) \subset \bigcap_{m \geqslant 0} \mathbb{S}_{\mathcal{U} ; T}\left(w_{m}\right)=\bigcap_{m \geqslant 0} \operatorname{cl}\left(\mathbb{S}_{\mathcal{U} ; T}\left(w_{m}\right)\right)=\{w\}$, that is, $w$ is an endpoint of $T$.

$\left(b_{3}\right)$ We show that wis an endpoint of $T$.

Indeed, let $\left(w_{m}: m \in\{0\} \cup \mathbb{N}\right) \in \mathcal{S}_{\mathcal{U}}\left(\mathbb{S}_{\mathcal{U} ; T}, x\right), x \in X$, and $w$ be such as in Step 12 . Analogously as in the proof of $\left(\mathrm{a}_{3}\right)$, we get $\forall_{m \in \mathbb{N}}\left\{T\left(\operatorname{cl}\left(\mathbb{S}_{\mathcal{U}_{i} T}\left(w_{m}\right)\right)\right) \subset \operatorname{cl}\left(\mathbb{S}_{\mathcal{U} ; T}\left(w_{m}\right)\right)\right\}$. Thus $T(w)=T\left(\bigcap_{m \geqslant 0} \operatorname{cl}\left(\mathbb{S}_{\mathcal{U} ; T}\left(w_{m}\right)\right)\right) \subset \bigcap_{m \geqslant 0} T\left(\operatorname{cl}\left(\mathbb{S}_{\mathcal{U} ; T}\left(w_{m}\right)\right)\right) \subset \bigcap_{m \geqslant 0} \operatorname{cl}\left(\mathbb{S}_{\mathcal{U} ; T}\left(w_{m}\right)\right)=\{w\}$, that is, $w$ is an endpoint of $T$.

\section{Examples, Remarks, and Comparisons of Our Results with the Well-Known Ones}

In this section we present some examples illustrating the concepts introduced so far. In Examples 7.1 and 7.2 we construct $\mathcal{\partial}$-families and $\mathcal{U}$-families, respectively.

Example 7.1. Let $L$ be an ordered normed space with cone $H \subset L$, let the family $D=\left\{p_{\alpha}\right.$ : $X \times X \rightarrow L, \alpha \in \mathcal{A}\}$ be a $D$-family, and let $(X, D)$ be a Hausdorff cone uniform space with cone $H$.

(A) The family $D$ is a 2 -family.

(B) Let both $X$ and $H$ contain at least two different points and let $H$ be normal with a normal constant $M$. Let $S_{1}=\{v, w\}, v \neq w$, be a subset of $X$ and, for each $\alpha \in \mathcal{A}$, let $c_{\alpha}, e_{\alpha} \in H$ be such that $c_{\alpha}>_{H} e_{\alpha}>_{H} 0$ and

$$
\forall_{\alpha \in \mathscr{A}} \forall_{x, y \in S_{1}}\left\{p_{\alpha}(x, y)+e_{\alpha} \prec_{H} c_{\alpha}\right\} .
$$

Let $\mathcal{\partial}=\left\{J_{\alpha}: X \times X \rightarrow L: \alpha \in \mathcal{A}\right\}$ be a family where, for each $\alpha \in \mathcal{A}, J_{\alpha}$ is defined by the formula

$$
J_{\alpha}(x, y)= \begin{cases}0, & \text { if } x=y=w, \\ p_{\alpha}(x, y)+e_{\alpha}, & \text { if }\{x, y\} \cap S_{1}=\{x, y\} \wedge \exists_{u \in\{x, y\}}\{u \neq w\}, \\ c_{\alpha,} & \text { if }\{x, y\} \cap S_{1} \neq\{x, y\}\end{cases}
$$

$x, y \in X$. We show that the family $\partial$ is a $\partial$-family on $X$.

Of course, condition (21) holds.

Now, we show that condition (22) holds. Indeed, let $x, y, z \in X$ be arbitrary and fixed. We consider three cases: (i) if $J_{\alpha}(x, z)=0$, then it is clear that $J_{\alpha}(x, z) \leq_{H} J_{\alpha}(x, y)+J_{\alpha}(y, z)$; (ii) if $J_{\alpha}(x, z)=p_{\alpha}(x, z)+e_{\alpha}$, then $\{x, z\} \cap S_{1}=\{x, z\} \wedge \exists_{u \in\{x, z\}}\{u \neq w\}$ which implies that $J_{\alpha}(x, y) \neq 0$ or $J_{\alpha}(y, z) \neq 0$ and, by (7.1), we get $J_{\alpha}(x, z) \leq_{H} J_{\alpha}(x, y)+J_{\alpha}(y, z)$; (iii) if $J_{\alpha}(x, z)=c_{\alpha}$, then $\{x, z\} \cap X \backslash S_{1} \neq \emptyset$ and, consequently, $J_{\alpha}(x, y)=c_{\alpha}$ or $J_{\alpha}(y, z)=c_{\alpha}$ which implies that $J_{\alpha}(x, z) \leq_{H} J_{\alpha}(x, y)+J_{\alpha}(y, z)$. Therefore, (22) holds. 
Now, let us observe that $\forall_{\alpha \in \mathcal{A}} \forall_{x, y \in X}\left\{p_{\alpha}(x, y)+e_{\alpha}-e_{\alpha}=p_{\alpha}(x, y) \in H\right\}$ which gives that $\forall_{\alpha \in \mathcal{A}} \forall_{x, y \in X}\left\{e_{\alpha} \leq_{H} p_{\alpha}(x, y)+e_{\alpha}\right\}$. Hence

$$
\forall_{\alpha \in \mathscr{A}} \forall_{x, y \in X}\left\{\left\|e_{\alpha}\right\| \leqslant M\left\|p_{\alpha}(x, y)+e_{\alpha}\right\|\right\}
$$

Next assume that the sequences $\left\{w_{m}\right\}$ and $\left\{v_{m}\right\}$ in $X$ satisfy (2.3) and (2.4). Then, in particular, from (2.4) we conclude that

$$
\forall_{\alpha \in \mathcal{A}} \forall_{0<\varepsilon<\left\|e_{\alpha}\right\| / M} \exists_{m_{0}=m_{0}(\alpha, \varepsilon) \in \mathbb{N}} \forall_{m \geqslant m_{0}}\left\{\left\|J_{\alpha}\left(w_{m}, v_{m}\right)\right\|<\varepsilon<\frac{\left\|e_{\alpha}\right\|}{M}\right\} .
$$

Hence, by (7.3),

$$
\forall_{\alpha \in \mathcal{A}} \forall_{m \geqslant m_{0}}\left\{\left\|J_{\alpha}\left(w_{m}, v_{m}\right)\right\|<\left\|p_{\alpha}\left(w_{m}, v_{m}\right)+e_{\alpha}\right\|\right\}
$$

Since $\forall_{\alpha \in \mathscr{A}}\left\{\left\|e_{\alpha}\right\| / M \leqslant\left\|c_{\alpha}\right\|\right\}$, condition (7.4) gives

$$
\forall_{\alpha \in \mathcal{A}} \forall_{m \geqslant m_{0}}\left\{\left\|J_{\alpha}\left(w_{m}, v_{m}\right)\right\|<\left\|c_{\alpha}\right\|\right\}
$$

By definition of a family 2 , from (7.5) and (7.6), denoting $m^{\prime}=\min \left\{m_{0}(\alpha, \varepsilon): \alpha \in \mathcal{A}\right\}$, we conclude that $\forall_{m \geqslant m^{\prime}}\left\{w_{m}=v_{m}=w\right\}$, which implies $\forall_{\alpha \in \mathscr{A}} \forall_{m \geqslant m^{\prime}}\left\{\left\|p_{\alpha}\left(w_{m}, v_{m}\right)\right\|=0\right\}$. In consequence, we obtain $\forall_{\alpha \in \mathcal{A}} \forall_{0<\varepsilon<\left\|e_{\alpha}\right\| / M} \exists_{m^{\prime} \in \mathbb{N}} \forall_{m \geqslant m^{\prime}}\left\{\left\|p_{\alpha}\left(w_{m}, v_{m}\right)\right\|=0<\varepsilon\right\}$. Thus, the sequences $\left\{w_{m}\right\}$ and $\left\{v_{m}\right\}$ satisfy (2.5). Therefore, the condition (23) holds.

Example 7.2. Let $(X, \Phi)$ be a Hausdorff uniform space with uniformity defined by a saturated family $\Phi=\left\{d_{\alpha}: \alpha \in \mathcal{A}\right\}$ of pseudometrics $d_{\alpha}: X \times X \rightarrow[0, \infty), \alpha \in \mathcal{A}$, uniformly continuous on $X^{2}$.

(A) The family $\boldsymbol{\Phi}$ is a $\mathcal{U}$-family.

(B) Let $X$ contain at least two different points. Let $S_{2} \subset X$, containing at least two different points, be arbitrary and fixed and let $\left\{c_{\alpha}\right\}_{\alpha \in \mathcal{A}}$ satisfy $\forall_{\alpha \in \mathcal{A}}\left\{\delta_{\alpha}\left(S_{2}\right)<c_{\alpha}\right\}$. Let $\mathcal{U}=\left\{U_{\alpha}: X \times X \rightarrow[0, \infty), \alpha \in \mathcal{A}\right\}$ be a family where, for each $\alpha \in \mathcal{A}, U_{\alpha}$ is defined by the formula

$$
U_{\alpha}(x, y)=\left\{\begin{array}{ll}
d_{\alpha}(x, y), & \text { if } S_{2} \cap\{x, y\}=\{x, y\}, \\
c_{\alpha}, & \text { if } S_{2} \cap\{x, y\} \neq\{x, y\},
\end{array} \quad x, y \in X\right.
$$

We show that the family $\mathcal{U}$ is a $\mathcal{U}$-family on $X$.

Indeed, we see that condition $(\mathcal{U} 1)$ does not hold only if there exist some $\alpha \in \mathcal{A}$ and $x, y, z \in X$ such that $U_{\alpha}(x, z)=c_{\alpha}, U_{\alpha}(x, y)=d_{\alpha}(x, y), U_{\alpha}(y, z)=d_{\alpha}(y, z)$, and $d_{\alpha}(x, y)+$ $d_{\alpha}(y, z)<c_{\alpha}$. However, then we conclude that there exists $v \in\{x, z\}$ such that $v \notin S_{2}$ and $x, y, z \in S_{2}$, which is impossible. Therefore, $\forall_{\alpha \in \mathscr{A}} \forall_{x, y, z \in X}\left\{U_{\alpha}(x, z) \leqslant U_{\alpha}(x, y)+U_{\alpha}(y, z)\right\}$, that is, condition $(\mathcal{U} 1)$ holds. 
Fixed Point Theory and Applications

To prove that $(\mathfrak{U} 2)$ holds we assume that the sequences $\left\{w_{m}\right\}$ and $\left\{v_{m}\right\}$ in $X$ satisfy (4.1) and (4.2). Then, in particular, (4.2) yields

$$
\forall_{\alpha \in \mathscr{A}} \forall_{0<\varepsilon<c_{\alpha}} \exists_{m_{0}=m_{0}(\alpha, \varepsilon) \in \mathbb{N}} \forall_{m \geqslant m_{0}}\left\{U_{\alpha}\left(w_{m}, v_{m}\right)<\varepsilon\right\} .
$$

By (7.8) and definition of $\mathcal{U}$, denoting $m^{\prime}=\min \left\{m_{0}(\alpha, \varepsilon): \alpha \in \mathcal{A}\right\}$, we conclude that

$$
\forall_{m \geqslant m^{\prime}}\left\{S_{2} \cap\left\{w_{m}, v_{m}\right\}=\left\{w_{m}, v_{m}\right\}\right\}
$$

From (7.9), the definition of $\mathcal{U}$ and (7.8), we get

$$
\forall_{\alpha \in \mathscr{A}} \forall_{0<\varepsilon<c_{\alpha}} \exists_{m^{\prime} \in \mathbb{N}} \forall_{m \geqslant m^{\prime}}\left\{d_{\alpha}\left(w_{m}, v_{m}\right)=0<\varepsilon\right\} .
$$

The result is that the sequences $\left\{w_{m}\right\}$ and $\left\{v_{m}\right\}$ satisfy (4.3). Therefore, condition (U2) holds. The following example illustrates Theorem 3.1(a) in cone metric space.

Example 7.3. Let $(L,\|\cdot\|), L=\mathbb{R}^{2}$, be a real normed space, let $H$ be a regular solid cone of the form $H=\{(x, y) \in L: x, y \geqslant 0\}$ and let $(X, P)$ be a cone metric space (see [27]) with a cone $H$ where $X=[0,1] \subset \mathbb{R}, P=\{p\}$, and $p: X \times X \rightarrow L$ is a cone metric of the form $p(x, y)=(|x-y|, 2|x-y|), x, y \in X$. Let $T: X \rightarrow 2^{X}$ be defined by

$$
T(x)= \begin{cases}{\left[\frac{1}{2}, 1\right],} & \text { if } x=0, \\ \{1\}, & \text { if } x \in\left(0, \frac{1}{2}\right) \cup\left(\frac{1}{2}, 1\right], \\ \{0,1\}, & \text { if } x=\frac{1}{2} .\end{cases}
$$

We note that for $q=1$, the map $T^{[q]}$ is closed in $X$.

Let $S_{1}=\{1 / 2,1\}$ and let $J: X \times X \rightarrow L$ be of the form

$$
J(x, y)= \begin{cases}(0,0), & \text { if } x=y=1, \\ p(x, y)+\left(\frac{1}{4}, \frac{1}{2}\right), & \text { if }\{x, y\} \cap S_{1}=\{x, y\} \wedge \exists_{u \in\{x, y\}}\{u \neq 1\}, \\ (2,2), & \text { if }\{x, y\} \cap S_{1} \neq\{x, y\}\end{cases}
$$

for $x, y \in X$. By Example 7.1(B), the family $\partial=\{J\}$ is a $\partial$-family. Now we define $\Omega=\{\omega\}$, $\omega: X \rightarrow L$, as follows:

$$
\omega(x)= \begin{cases}\left(1, \frac{3}{2}\right), & \text { for } x \in[0,1) \backslash\left\{\frac{1}{2}\right\} \\ (2,2), & \text { for } x=\frac{1}{2} \\ (0,0), & \text { for } x=1\end{cases}
$$

Of course, $\forall_{x \in X}\left\{0 \preceq_{H} \omega(x)\right\}$ and $\omega$ is not lsc on $X$. 
(a) The map $T$ is weak $\left(2, \Omega ; X_{0}\right)$-dissipative on $X$ where $\Omega=\{\omega\}$ and $X_{0}=\{1 / 2,1\}$.

Indeed, if $x=1$, then, by definition $T$, a sequence $\left(w_{m}: m \in\{0\} \cup \mathbb{N}\right)$ satisfying (1.1) is of the form $w_{m}=1$ where $m \in\{0\} \cup \mathbb{N}$ and satisfies $\forall_{m \in\{0\} \cup \mathbb{N}}\left\{J\left(w_{m}, w_{m+1}\right)=(0,0) \leq_{H} \omega(1)-\right.$ $\left.\omega(1)=\omega\left(w_{m}\right)-\omega\left(w_{m+1}\right)=(0,0)\right\}$, that is, $(2.7)$ holds.

If $x=1 / 2$, then there exists a sequence $\left(w_{m}: m \in\{0\} \cup \mathbb{N}\right)$ satisfying (1.1) of the form $w_{0}=1 / 2, w_{m}=1$ where $m \in \mathbb{N}$ and satisfies $J\left(w_{0}, w_{1}\right)=J(1 / 2,1)=(3 / 4,3 / 2) \leq_{H}(2,2)-$ $(0,0)=\omega\left(w_{0}\right)-\omega\left(w_{1}\right)$ and $\forall_{m \in \mathbb{N}}\left\{J\left(w_{m}, w_{m+1}\right)=(0,0) \leq_{H} \omega(1)-\omega(1)=\omega\left(w_{m}\right)-\omega\left(w_{m+1}\right)=\right.$ $(0,0)\}$, that is, $(2.7)$ holds.

Consequently, $\{1 / 2,1\} \subset X_{0}$.

We see that $X_{0}=\{1 / 2,1\}$. Otherwise, $X_{0} \backslash\{1 / 2,1\} \neq \emptyset$ and the following two cases hold:

Case 1. If $x=0$, then, by definition of $T$, for each sequence $\left(w_{m}: m \in\{0\} \cup \mathbb{N}\right)$ satisfying (1.1) we have that $w_{0}=0, w_{1} \in[1 / 2,1]$ and then, by definition of $J$,

$$
J\left(w_{0}, w_{1}\right)=J\left(0, w_{1}\right)=(2,2)>_{H} \omega(0)-\omega\left(w_{1}\right)= \begin{cases}(0,0) & \text { if } w_{1} \in\left(\frac{1}{2}, 1\right) \\ \left(1, \frac{3}{2}\right) & \text { if } w_{1}=1 \\ -\left(1, \frac{1}{2}\right) & \text { if } w_{1}=\frac{1}{2}\end{cases}
$$

which means that (2.7) does not hold.

Case 2. If $x \notin\{0,1 / 2,1\}$, then, by definition of $T$, each sequence $\left(w_{m}: m \in\{0\} \cup \mathbb{N}\right)$ satisfying (1.1) is of the form $w_{0}=x$ and, for each $m \in \mathbb{N}, w_{m}=1$. Next, by definition of $J$, since $x \notin S_{1}$, we obtain

$$
J\left(w_{0}, w_{1}\right)=J(x, 1)=(2,2)>_{H} \omega\left(w_{0}\right)-\omega\left(w_{1}\right)=\left(1, \frac{3}{2}\right),
$$

which means that (2.7) does not hold.

Consequently, $X_{0}=\{1 / 2,1\}$.

(b) All assumptions of Theorem 3.1(a) hold, and $1 \in X_{0}$ is the periodic point of $T$, that is, $1 \in \operatorname{Fix}(T)$.

Remark 7.4. Let $L, H,(X, D), T$ and 2-family be such as in Example 7.3.

(i) We see that for this 2 -family the map $T$ is not $(2, \Omega)$-dissipative on $X$ for any $\Omega$ (consequently, by Remark 2.9(ii), for any $\Omega, T$ is not strictly $(2, \Omega)$-dissipative on $X)$. Indeed, suppose that there exists $\Omega=\{\omega\}$ such that $\omega: X \rightarrow L$ is a map satisfying $\forall_{x \in X}\left\{0 \preceq_{H} \omega(x)\right\}$ and such that $T$ is $(2, \Omega)$-dissipative on $X$. Then, in particular, for a dynamic process $w_{0}=1 / 2, w_{1}=0, w_{2}=1 / 2, w_{3}=0$, and $w_{m}=1$ for $m \geqslant 4$, by (2.7), we must have $(0,0) \prec_{H}(2,2)=J\left(w_{0}, w_{1}\right)=J(1 / 2,0) \leq_{H} \omega(1 / 2)-$ $\omega(0)$ so $\omega(0) \prec_{H} \omega(1 / 2)$ and $(0,0) \prec_{H}(2,2)=J\left(w_{1}, w_{2}\right)=J(0,1 / 2) \preceq_{H} \omega(0)-\omega(1 / 2)$ so $\omega(1 / 2) \prec_{H} \omega(0)$, which are contradictions. 
(ii) We see that $X_{0}=\{1 / 2,1\}$ and $T\left(X_{0}\right)=\{0,1\} \neq X_{0}$.

In Examples 7.5 and 7.7 we illustrate Theorem 5.3(a) for dissipative set-valued and single-valued dynamic systems, respectively.

Example 7.5. Let $(X, d)$ be a metric space, where $X=[0,1 / 2] \cup\{3 / 4,1\}$ and $d: X \times X \rightarrow[0, \infty)$ is a metric of the form $d(x, y)=|x-y|, x, y \in X$. Let $T: X \rightarrow 2^{X}$ be of the form

$$
T(x)= \begin{cases}\{1\}, & \text { for } x=0 \\ {\left[0, \frac{1}{2} x\right],} & \text { for } x \in\left(0, \frac{1}{2}\right] \\ \{0\}, & \text { for } x=\frac{3}{4} \\ \left\{\frac{3}{4}\right\}, & \text { for } x=1\end{cases}
$$

Let $S_{2}=[0,1 / 2]$. By Example 7.2(B), the family $u=\{U: X \times X \rightarrow[0, \infty)\}$, where

$$
U(x, y)=\left\{\begin{array}{ll}
d(x, y), & \text { if } S_{2} \cap\{x, y\}=\{x, y\}, \\
2, & \text { if } S_{2} \cap\{x, y\} \neq\{x, y\},
\end{array} \quad x, y \in X\right.
$$

is a $\mathcal{U}$-family on $X$. We observe that

$$
\begin{aligned}
& T^{[2]}(x)= \begin{cases}\left\{\frac{3}{4}\right\}, & \text { for } x=0, \\
{\left[0, \frac{1}{4} x\right] \cup\{1\},} & \text { for } x \in\left(0, \frac{1}{2}\right], \\
\{1\}, & \text { for } x=\frac{3}{4}, \\
\{0\}, & \text { for } x=1,\end{cases} \\
& T^{[3]}(x)= \begin{cases}\{0\}, & \text { for } x=0, \\
{\left[0, \frac{1}{8} x\right] \cup\left\{\frac{3}{4}, 1\right\},} & \text { for } x \in\left(0, \frac{1}{2}\right], \\
\left\{\frac{3}{4}\right\}, & \text { for } x=\frac{3}{4}, \\
\{1\}, & \text { for } x=1,\end{cases}
\end{aligned}
$$




$$
T^{[4]}(x)= \begin{cases}\{1\}, & \text { for } x=0, \\ \left\{0, \frac{1}{16} x\right] \cup\left\{\frac{3}{4}, 1\right\}, & \text { for } x \in\left(0, \frac{1}{2}\right] \\ \{0\}, & \text { for } x=\frac{3}{4} \\ \left\{\frac{3}{4}\right\}, & \text { for } x=1,\end{cases}
$$

so, the map $T^{[3]}$ is closed in $X$. However, the map $T^{[4]}$ is not closed in $X$.

Let $\Gamma=\{\gamma\}, \gamma: X \rightarrow[0, \infty)$, be of the form $\gamma(x)=x, x \in X$.

(a) $T$ is weak $\left(\mathcal{U}, \Gamma ; X_{0}\right)$-dissipative on $X$, where $X_{0}=(0,1 / 2]$. Indeed, let $x \in(0,1 / 2]$ be arbitrary and fixed, then there exists a dynamic process $\left(w_{m}: m \in\{0\} \cup \mathbb{N}\right)$ given by the formula $w_{0}=x, w_{m}=\left(1 / 2^{m}\right) x, m \in \mathbb{N}$, such that $U\left(w_{0}, w_{1}\right)=d(x,(1 / 2) x)=$ $x / 2 \leqslant x-x / 2=\gamma\left(w_{0}\right)-\gamma\left(w_{1}\right) ; \forall_{m \in \mathbb{N}}\left\{U\left(w_{m}, w_{m+1}\right)=d\left(w_{m}, w_{m+1}\right) \leqslant\left(1 / 2^{m}\right) x-\right.$ $\left.\left(1 / 2^{m+1}\right) x=\gamma\left(w_{m}\right)-\gamma\left(w_{m+1}\right)\right\}$. This implies that the dynamic process $\left(w_{m}: m \in\right.$ $\{0\} \cup \mathbb{N}$ ) satisfies $(1.1)$ and (4.4). Consequently, $(0,1 / 2] \subset X_{0}$. The fact that $X_{0} \backslash$ $(0,1 / 2]=\emptyset$ follows from considerations in the remark below.

(b) The $0 \in \operatorname{cl} X_{0}$ is the periodic point of $T(q=3)$.

Remark 7.6. Let $X, \boldsymbol{\Phi}, T$, and $\mathcal{U}$-family be such as in Example 7.5. We see that for this $\mathcal{U}$-family the map $T$ is not $(\mathcal{U}, \Gamma)$-dissipative on $X$ for any $\Gamma$ (consequently, by Remark 2.9(ii), for any $\Gamma, T$ is not strictly $(\mathcal{U}, \Gamma)$-dissipative on $X)$. Indeed, suppose that there exists $\Gamma=\{\gamma\}$ such that $\gamma: X \rightarrow[0, \infty]$ and that $T$ is $(\mathcal{U}, \Gamma)$-dissipative on $X$. Then, we have a unique dynamic process $\left(w_{m}: m \in\{0\} \cup \mathbb{N}\right)$ starting at $w_{0}=3 / 4$ which is defined by $w_{1}=0 \in T\left(w_{0}\right), w_{2}=1 \in T\left(w_{1}\right)$, $w_{3 m+1}=0 \in T\left(w_{3 m}\right), w_{3 m+2}=1 \in T\left(w_{3 m+1}\right)$, and $w_{3 m}=3 / 4 \in T\left(w_{3 m-1}\right)$ for $m \in \mathbb{N}$ and for this process we have $0<U\left(w_{0}, w_{1}\right)=2 \leqslant \gamma\left(w_{0}\right)-\gamma\left(w_{1}\right), 0<U\left(w_{1}, w_{2}\right)=2 \leqslant \gamma\left(w_{1}\right)-\gamma\left(w_{2}\right)$, and $0<U\left(w_{2}, w_{3}\right)=2 \leqslant \gamma\left(w_{2}\right)-\gamma\left(w_{3}\right)=\gamma\left(w_{2}\right)-\gamma\left(w_{0}\right)$. Hence $\gamma\left(w_{0}\right)<\gamma\left(w_{2}\right)<\gamma\left(w_{1}\right)<\gamma\left(w_{0}\right)$, which is impossible.

Example 7.7. Let $(X, d)$ be a metric space, where $X=[0,1]$ and $d: X \times X \rightarrow[0, \infty)$ is a metric of the form $d(x, y)=|x-y|, x, y \in X$. Let $T: X \rightarrow X$ be of the form

$$
T(x)= \begin{cases}1, & \text { for } x \in\left[0, \frac{1}{8}\right], \\ -2 x+\frac{5}{4}, & \text { for } x \in\left(\frac{1}{8}, \frac{3}{8}\right), \\ \frac{1}{2}, & \text { for } x \in\left[\frac{3}{8}, \frac{5}{8}\right], \\ -2 x+\frac{7}{4}, & \text { for } x \in\left(\frac{5}{8}, \frac{7}{8}\right), \\ 0, & \text { for } x \in\left[\frac{7}{8}, 1\right] .\end{cases}
$$


By Example 7.2(A), the family $\mathcal{U}=\{U: X \times X \rightarrow[0, \infty)\}$, where $U(x, y)=d(x, y), x, y \in X$, is $U$-family on $X$.

Let $\Gamma=\{\gamma\}, \gamma: X \rightarrow[0, \infty)$, be of the form

$$
r(x)= \begin{cases}1, & \text { for } x \in\left[0, \frac{5}{16}\right) \cup\left(\frac{11}{16}, 1\right], \\ 3, & \text { for } x \in\left[\frac{5}{16}, \frac{3}{8}\right) \cup\left(\frac{5}{8}, \frac{11}{16}\right], \\ 2, & \text { for } x \in\left[\frac{3}{8}, \frac{1}{2}\right) \cup\left(\frac{1}{2}, \frac{5}{8}\right], \\ 0, & \text { for } x=\frac{1}{2}\end{cases}
$$

(a) We show that $T$ is weak $\left(\mathcal{U}, \Gamma ; X_{0}\right)$-dissipative on $X$, where $X_{0}=[5 / 16,11 / 16]$. Indeed, let $x \in X_{0}$ be arbitrary and fixed. We consider the following four cases.

Case 1. If $x=1 / 2$, then a dynamic process $\left(w_{m}: m \in\{0\} \cup \mathbb{N}\right)$ of $(X, T)$ starting at $x$ is of the form $w_{m}=1 / 2, m \in\{0\} \cup \mathbb{N}$. Consequently, $\forall_{m \in\{0\} \cup \mathbb{N}}\left\{U\left(w_{m}, w_{m+1}\right)=d\left(w_{m}, w_{m+1}\right)=0 \leqslant\right.$ $\left.0-0=\gamma\left(w_{m}\right)-\gamma\left(w_{m+1}\right)\right\}$, that is, (4.4) holds.

Case 2. If $x \in[5 / 16,3 / 8)$, then a dynamic process $\left(w_{m}: m \in\{0\} \cup \mathbb{N}\right)$ of $(X, T)$ starting at $x$ is of the form $w_{0}=x, w_{1}=-2 x+5 / 4 \in(1 / 2,5 / 8]$, and $w_{m}=1 / 2, m \geqslant 2$. Therefore, $U\left(w_{0}, w_{1}\right)=$ $d\left(w_{0}, w_{1}\right) \leqslant 1=3-2=\gamma\left(w_{0}\right)-\gamma\left(w_{1}\right), U\left(w_{1}, w_{2}\right)=d\left(w_{1}, w_{2}\right) \leqslant 2-0=\gamma\left(w_{1}\right)-\gamma\left(w_{2}\right)$, and $\forall_{m \geqslant 2}\left\{U\left(w_{m}, w_{m+1}\right)=d\left(w_{m}, w_{m+1}\right)=0 \leqslant 0-0=\gamma\left(w_{m}\right)-\gamma\left(w_{m+1}\right)\right\}$, that is, (4.4) holds.

Case 3. If $x \in[3 / 8,1 / 2) \cup(1 / 2,5 / 8]$, then a dynamic process $\left(w_{m}: m \in\{0\} \cup \mathbb{N}\right)$ of $(X, T)$ starting at $x$ is of the form $w_{0}=x$ and $w_{m}=1 / 2$ for $m \in \mathbb{N}$. Moreover, (4.4) holds since $U\left(w_{0}, w_{1}\right)=d\left(w_{0}, w_{1}\right) \leqslant 2-0=\gamma\left(w_{0}\right)-\gamma\left(w_{1}\right)$ and $\forall_{m \in \mathbb{N}}\left\{U\left(w_{m}, w_{m+1}\right)=d\left(w_{m}, w_{m+1}\right)=0 \leqslant\right.$ $\left.0-0=\gamma\left(w_{m}\right)-\gamma\left(w_{m+1}\right)\right\}$.

Case 4. If $x \in(5 / 8,11 / 16]$, then a dynamic process $\left(w_{m}: m \in\{0\} \cup \mathbb{N}\right)$ of $(X, T)$ starting at $x$ is of the form $w_{0}=x, w_{1}=-2 x+7 / 4 \in[3 / 8,1 / 2)$, and $w_{m}=1 / 2, m \geqslant 2$. We also get that $U\left(w_{0}, w_{1}\right)=d\left(w_{0}, w_{1}\right) \leqslant 1=3-2=\gamma\left(w_{0}\right)-\gamma\left(w_{1}\right), U\left(w_{1}, w_{2}\right)=d\left(w_{1}, w_{2}\right) \leqslant 2-0=$ $\gamma\left(w_{1}\right)-\gamma\left(w_{2}\right)$, and $\forall_{m \geqslant 2}\left\{U\left(w_{m}, w_{m+1}\right)=d\left(w_{m}, w_{m+1}\right)=0 \leqslant 0-0=\gamma\left(w_{m}\right)-\gamma\left(w_{m+1}\right)\right\}$.

(b) Now, we show that if $x \in[0,5 / 16) \cup(11 / 16,1]$, then $x \notin X_{0}$. Indeed, let $x \in[0,5 / 16)$ and let $\left(w_{m}: m \in\{0\} \cup \mathbb{N}\right)$ be a dynamic process of $(X, T)$ starting at $x$. Then $w_{0}=x$, $w_{1} \in(5 / 8,1]$, and

$$
U\left(w_{0}, w_{1}\right)=d\left(w_{0}, w_{1}\right)> \begin{cases}-2=1-3=\gamma\left(w_{0}\right)-\omega\left(w_{1}\right), & \text { if } w_{1} \in\left(\frac{5}{8}, \frac{11}{16}\right] \\ 0=1-1=\gamma\left(w_{0}\right)-\omega\left(w_{1}\right), & \text { if } w_{1} \in\left(\frac{11}{16}, 1\right] .\end{cases}
$$


Now, let $x \in(11 / 16,1]$ and let $\left(w_{m}: m \in\{0\} \cup \mathbb{N}\right)$ be a dynamic process of $(X, T)$ starting at $x$. Then $w_{0}=x, w_{1} \in[0,3 / 8)$, and

$$
U\left(w_{0}, w_{1}\right)=d\left(w_{0}, w_{1}\right)> \begin{cases}0=1-1=\gamma\left(w_{0}\right)-\omega\left(w_{1}\right), & \text { if } w_{1} \in\left[0, \frac{5}{16}\right), \\ -2=1-3=\gamma\left(w_{0}\right)-\omega\left(w_{1}\right), & \text { if } w_{1} \in\left[\frac{5}{16}, \frac{3}{8}\right) .\end{cases}
$$

Consequently, $T$ is weak $\left(\mathcal{U}, \Gamma ; X_{0}\right)$-dissipative on $X$, where $X_{0}=[5 / 16,11 / 16]$.

(c) The map $T$ is closed on $X, 1 / 2 \in \operatorname{Fix}(T)$, and $1 / 2 \in X_{0}$.

Remark 7.8. Let $X, \boldsymbol{\oplus}, T$ and $\mathcal{U}$-family be such as in Example 7.7. We see that for this $\mathcal{U}$-family the map $T$ is not $(\mathcal{U}, \Gamma)$-dissipative on $X$ for any $\Gamma$; see $(b)$.

The following example shows that in Theorem 5.3 for the existence of periodic points the assumption that the map $T^{[q]}$ is closed in $X$ for some $q \in \mathbb{N}$ is essential.

Example 7.9. Let $(X, d)$ be a metric space, where $X=[0,1]$ and $d: X \times X \rightarrow[0, \infty)$ is a metric of the form $d(x, y)=|x-y|, x, y \in X$. Let $T: X \rightarrow 2^{X}$ be of the form

$$
T(x)= \begin{cases}\left(\frac{1}{2}, 1\right], & \text { if } x=0 \\ \left(\frac{1}{2^{m+1}}, \frac{1}{2^{m}}\right], & \text { if } x \in\left(\frac{1}{2^{m}}, \frac{1}{2^{m-1}}\right], m \in \mathbb{N}\end{cases}
$$

let $\mathcal{U}=\{d\}$ and let $\Gamma=\{\gamma\}, \gamma: X \rightarrow[0, \infty)$, be of the form

$$
\gamma(x)= \begin{cases}4, & \text { if } x \in 0 \\ x, & \text { if } x \in(0,1] .\end{cases}
$$

We observe the following.

(a) $T$ is $(\mathcal{U}, \Gamma)$-dissipative and strictly $(\mathcal{U}, \Gamma)$-dissipative on $X$.

(b) For each $x \in X, \mathcal{W}_{\mathcal{U}}(T, x)=\mathcal{S}_{\mathfrak{U}}(T, x)$, and $\left\{\{0\}=\left\{w: \lim _{m} d\left(w_{m}, w\right)=0 \wedge\left(w_{m}\right.\right.\right.$ : $\left.m \in\{0\} \cup \mathbb{N}) \in S_{\mathfrak{U}}(T, x)\right\}$.

(c) For each $q \in \mathbb{N}$, the map $T^{[q]}$ is not closed in $X$.

(d) The map $T$ does not have periodic points in $X$.

The following example illustrates Theorem 6.2 $\left(\mathrm{a}_{1}\right)$.

Example 7.10. Let $(X, d)$ be a metric space, where $X=[0,1]$ and $d: X \times X \rightarrow[0, \infty)$ is a metric of the form $d(x, y)=|x-y|, x, y \in X$. 
Fixed Point Theory and Applications

Let $S_{2}=[0,1 / 2]$ and let $U: X \times X \rightarrow[0, \infty)$ be of the form

$$
U(x, y)=\left\{\begin{array}{ll}
d(x, y), & \text { if } S_{2} \cap\{x, y\}=\{x, y\}, \\
2, & \text { if } S_{2} \cap\{x, y\} \neq\{x, y\},
\end{array} \quad x, y \in X\right.
$$

By Example 7.2(B), the family $\mathcal{U}=\{U\}$ is a $\mathcal{U}$-family. Define $\Gamma=\{\gamma\}, \gamma: X \rightarrow[0, \infty]$, as follows:

$$
r(x)= \begin{cases}x, & \text { if } x \in\left[0, \frac{1}{2}\right], \\ 6, & \text { if } x \in\left(\frac{1}{2}, \frac{3}{4}\right], \quad x \in X . \\ 4, & \text { if } x \in\left(\frac{3}{4}, 1\right) \\ 8, & \text { if } x=1\end{cases}
$$

Let $T: X \rightarrow 2^{X}$ be of the form

$$
T(x)= \begin{cases}\{0\}, & \text { if } x=0 \\ \left\{\frac{1}{n+1}\right\}, & \text { if } x=\frac{1}{n} \wedge n \geqslant 2 \\ \left\{\frac{1}{n+1}\right\}, & \text { if } x \in\left(\frac{1}{n+1}, \frac{1}{n}\right) \wedge n \geqslant 2 \\ \left\{\frac{7}{8}\right\}, & \text { if } x \in\left(\frac{1}{2}, \frac{3}{4}\right) \\ \left.\frac{7}{8}, 1\right), & \text { if } x=\frac{3}{4}, \\ \left\{\frac{1}{2}\right\}, & \text { if } x \in\left(\frac{3}{4}, 1\right) \\ \left(\frac{1}{2}, \frac{3}{4}\right), & \text { if } x=1\end{cases}
$$

We observe that

(a) $T$ is $(\mathcal{U}, \Gamma)$-dissipative on $X$. Indeed, let $x \in X$ be arbitrary and fixed. We consider seven cases

Case 1. If $x=0$, then each dynamic process starting at $w_{0}=0$ is of the form $\forall_{m \in \mathbb{N}}\left\{w_{m}=0 \in\right.$ $\left.T\left(w_{m-1}\right)\right\}$ and $\forall_{m \in\{0\} \cup \mathbb{N}}\left\{U\left(w_{m}, w_{m+1}\right)=0 \leqslant \gamma\left(w_{m}\right)-\gamma\left(w_{m+1}\right)\right\}$.

Case 2. If $x \in(0,1 / 2) \backslash\{1 / n: n \geqslant 3\}$, then there exists $l_{0} \geqslant 2$ such that $x \in\left(1 /\left(l_{0}+1\right), 1 / l_{0}\right)$ and each dynamic process starting at $w_{0}=x$ is of the form $\forall_{m \geqslant 1}\left\{w_{m}=1 /\left(l_{0}+m\right)\right\}$. Therefore $\left.U\left(w_{0}, w_{1}\right)=d\left(x, 1 /\left(l_{0}+1\right)\right)=x-1 /\left(l_{0}+1\right) \leqslant \gamma(x)-\gamma\left(w_{l_{0}+1}\right)=\gamma\left(w_{0}\right)-\gamma\left(w_{1}\right)\right\}$ and $\forall_{m \in \mathbb{N}}\left\{U\left(w_{m}, w_{m+1}\right)=1 /\left(l_{0}+m\right)-1 /\left(l_{0}+m+1\right) \leqslant \gamma\left(w_{m}\right)-\gamma\left(w_{m+1}\right)\right\}$. 
Case 3. If $x \in\{1 / n: n \geqslant 2\}$, then there exists $l_{0} \geqslant 2$ such that $x=1 / l_{0}$ and each dynamic process starting at $w_{0}=x$ is of the form $\forall_{m \geqslant 1}\left\{w_{m}=1 /\left(l_{0}+m\right)\right\}$. Therefore $\forall_{m \in\{0\} \cup \mathbb{N}}\left\{U\left(w_{m}, w_{m+1}\right)=1 /\left(l_{0}+m\right)-1 /\left(l_{0}+m+1\right) \leqslant \gamma\left(w_{m}\right)-\gamma\left(w_{m+1}\right)\right\}$.

Case 4. If $x \in(1 / 2,3 / 4)$, then each dynamic process starting at $w_{0}=x$ is of the form $w_{1}=7 / 8$ and $\forall_{m \geqslant 2}\left\{w_{m}=1 / m\right\}$. Therefore $U\left(w_{0}, w_{1}\right)=2 \leqslant 6-4=\gamma\left(w_{0}\right)-\gamma\left(w_{1}\right), U\left(w_{1}, w_{2}\right)=2 \leqslant$ $4-1 / 2=\gamma\left(w_{0}\right)-\gamma\left(w_{1}\right)$ and $\forall_{m \geqslant 2}\left\{U\left(w_{m}, w_{m+1}\right)=1 / m-1 /(m+1) \leqslant \gamma\left(w_{m}\right)-\gamma\left(w_{m+1}\right)\right\}$.

Case 5. If $x=3 / 4$, then each dynamic process starting at $w_{0}=x$ is of the form $w_{1} \in[7 / 8,1)$ and $\forall_{m \geqslant 2}\left\{w_{m}=1 / m\right\}$. Therefore $U\left(w_{0}, w_{1}\right)=2 \leqslant 6-4=\gamma\left(w_{0}\right)-\gamma\left(w_{1}\right), U\left(w_{1}, w_{2}\right)=2 \leqslant$ $4-1 / 2=\gamma\left(w_{0}\right)-\gamma\left(w_{1}\right)$, and $\forall_{m \geqslant 2}\left\{U\left(w_{m}, w_{m+1}\right)=1 / m-1 /(m+1) \leqslant \gamma\left(w_{m}\right)-\gamma\left(w_{m+1}\right)\right\}$.

Case 6. If $x \in(3 / 4,1)$, then each dynamic process starting at $w_{0}=x$ is of the form $\forall_{m \geqslant 1}\left\{w_{m}=\right.$ $1 /(m+1)\}$. Therefore, $U\left(w_{0}, w_{1}\right)=2 \leqslant 4-1 / 2=\gamma\left(w_{0}\right)-\gamma\left(w_{1}\right)$ and $\forall_{m \geqslant 2}\left\{U\left(w_{m-1}, w_{m}\right)=\right.$ $\left.1 / m-1 /(m+1) \leqslant \gamma\left(w_{m-1}\right)-\gamma\left(w_{m}\right)\right\}$.

Case 7. If $x=1$, then each dynamic process starting at $w_{0}=x$ is of the form $w_{1} \in(1 / 2,3 / 4)$, $w_{2}=7 / 8, w_{3}=1 / 2$, and $\forall_{m \geqslant 4}\left\{w_{m}=1 /(m-1)\right\}$. Therefore $U\left(w_{0}, w_{1}\right)=2 \leqslant 8-6=\gamma\left(w_{0}\right)-$ $\gamma\left(w_{1}\right), U\left(w_{1}, w_{2}\right)=2 \leqslant 6-4=\gamma\left(w_{0}\right)-\gamma\left(w_{1}\right)$ and by analogous argumentation as in Case 4 we obtain that (1.1) and (4.4) hold in this case.

Consequently, $T$ is $(\mathcal{U}, \Gamma)$-dissipative on $X$.

(b) $\mathbb{W}_{\mathcal{U}_{; T}}$ is of the form

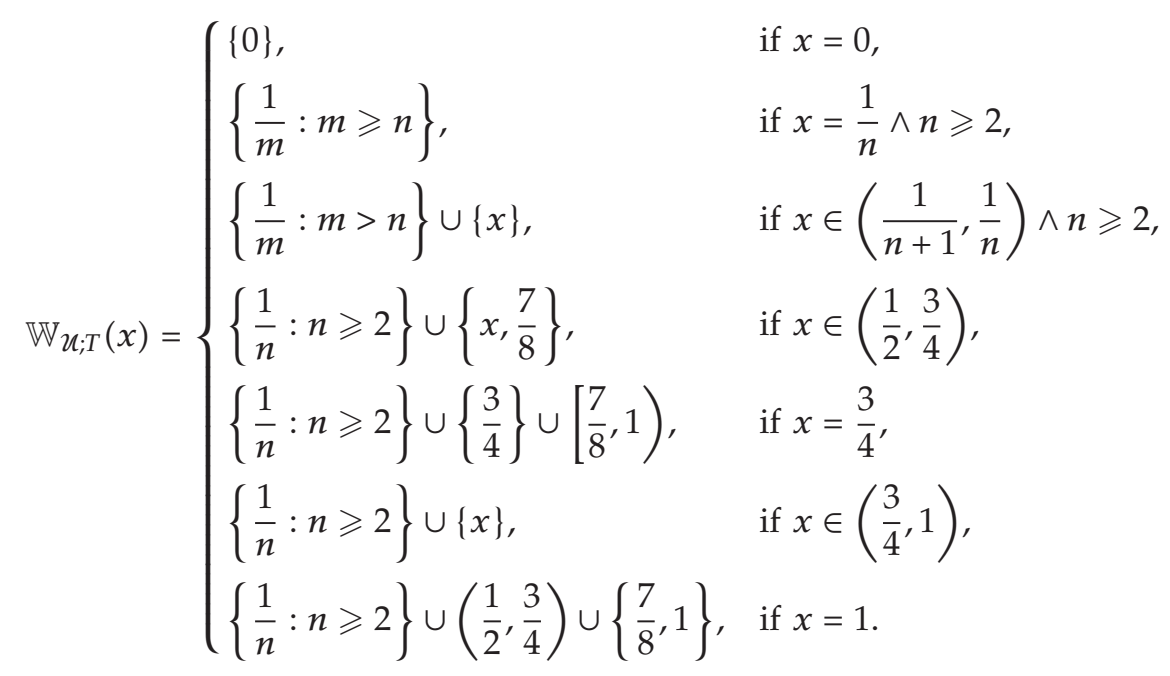

(c) $\mathbb{W}_{\mathfrak{U} ; T}$ is $(\mathcal{U}, \Gamma)$-dissipative on $X$.

(d) For each $x \in X$, there exist $\left(w_{m}: m \in\{0\} \cup \mathbb{N}\right) \in \mathcal{W}_{\mathfrak{u}}\left(\mathbb{W}_{\mathfrak{U}_{i T}}, x\right)$ and $w=0 \in X$ such that $\left(w_{m}: m \in\{0\} \cup \mathbb{N}\right)$ converges to $w$ and $\bigcap_{m \geqslant 2} \mathbb{W}_{\mathcal{u} ; T}\left(w_{m}\right)=\{w\}=\{0\}$. We see that $w=0$ is an endpoint of $T$ in $X$.

The assertions $\left(a_{1}\right)$ of the Theorem 6.2 hold. 
Remark 7.11. It is worth noticing that in Example 7.10, there exists $x \in X$ such that $\mathbb{W}_{\mathfrak{U}_{T T}}(x)$ is not closed. Indeed, $\mathbb{W}_{\mathcal{U}_{i}}(1 / 2)=\{1 / m: m \geqslant 2\}$ is not closed. Moreover, $T$ is not lsc on $X$. Consequently, the assumptions of Theorems 6.2( $\left.\mathrm{a}_{2}\right)$ and $6.2\left(\mathrm{a}_{3}\right)$ do not hold.

The following example illustrates Theorems 6.2( $\left(\mathrm{a}_{2}\right)$ and 6.2( $\left(\mathrm{a}_{3}\right)$.

Example 7.12. Let $(X, d)$ be a metric space, where $X=[0,1 / 2], d(x, y)=|x-y|, x, y \in X$, and $u=\{d\}$. Let $T: X \rightarrow 2^{X}$ be of the form $T(x)=[0,(1 / 2) x], x \in X$, and let $\Gamma=\{\gamma: X \rightarrow$ $[0, \infty)\}$ be defined as follows $\gamma(x)=x, x \in X$.

(a) The map $T$ is $(\mathcal{U}, \Gamma)$-dissipative. Indeed, if $x \in X$ is arbitrary and fixed, then each dynamic process $\left(w_{m}: m \in\{0\} \cup \mathbb{N}\right)$ starting at $w_{0}=x$ is of the form $w_{m} \in T\left(w_{m-1}\right)=$ $\left[0,(1 / 2) w_{m-1}\right]$ for $m \in \mathbb{N}$. Therefore, we have $\forall_{m \in\{0\} \cup \mathbb{N}}\left\{0<U\left(w_{m}, w_{m+1}\right)=w_{m}-\right.$ $\left.w_{m+1}=\gamma\left(w_{m}\right)-\gamma\left(w_{m+1}\right)\right\}$. Thus conditions (1.1) and (4.4) hold.

(b) We observe that

$$
\mathbb{W}_{\mathcal{U} ; T}(x)= \begin{cases}\{0\}, & \text { if } x \in 0, \\ {\left[0, \frac{1}{2} x\right],} & \text { if } x \in(0,1]\end{cases}
$$

and $\mathbb{W}_{\mathcal{U} ; T}$ is $(\mathcal{U}, \Gamma)$-dissipative on $X$.

(c) For each $x \in X$, the set $\mathbb{W}_{\mathcal{U} ; T}(x)$ is closed.

(d) The assertions of Theorem 6.2( $\left.\mathrm{a}_{2}\right)$ hold.

(e) The map $\mathbb{W}_{\mathcal{U} ; T}$ is closed.

(f) We have that $w=0 \in \operatorname{End}(T)$ and $\bigcap_{m \geqslant 0} \operatorname{cl}\left(\mathbb{W}_{\mathcal{U}_{i T}}\left(w_{m}\right)\right)=\{w\}$.

(g) The map $T$ is lsc on $X$.

(h) The assertions $\left(a_{3}\right)$ hold.

(i) The map $T$ is not strictly $(\mathcal{U}, \Gamma)$-dissipative. Indeed, if $x=1 / 2 \in X$, then we have that $T^{[m]}(x)=\left[0,\left(1 / 2^{m}\right) x\right]$ for $m \in \mathbb{N}$ and a generalized sequence of iterations $\left(w_{m}: m \in\{0\} \cup \mathbb{N}\right)$ starting at $w_{0}=x$, of the form $w_{1}=0, w_{2}=1 / 8$ and $w_{m}=0$ for $m \geqslant 3$, not satisfies $(4.4)$ since $U\left(w_{1}, w_{2}\right)=1 / 8>-1 / 8=w_{1}-w_{2}=\gamma\left(w_{1}\right)-\gamma\left(w_{2}\right)$.

The following example illustrates Theorem $6.2\left(\mathrm{a}_{2}\right)$.

Example 7.13. Let $(X, d), X=[0,1]$, and let $\mathcal{U}=\{d\}$. Let $T: X \rightarrow 2^{X}$ be of the form

$$
T(x)= \begin{cases}\{1\}, & \text { if } x=0 \\ {\left[0, \frac{1}{2} x\right],} & \text { if } x \in(0,1) \\ \{1\}, & \text { if } x=1\end{cases}
$$


and let $\Gamma=\{\gamma: X \rightarrow[0, \infty)\}$ where $\gamma$ is of the form

$$
r(x)= \begin{cases}1, & \text { if } x=0 \\ x, & \text { if } x \in(0,1), x \in X \\ 0, & \text { if } x=1\end{cases}
$$

(a) The map $T$ is $(\mathcal{U}, \Gamma)$-dissipative on $X$. Indeed, let $x \in X$ be arbitrary and fixed. We consider three cases.

Case 1. If $x=0$, then a dynamic process starting at $w_{0}=0$ is of the form $\forall_{m \in \mathbb{N}}\left\{w_{m}=1 \in\right.$ $\left.T\left(w_{m-1}\right)\right\}$ and $U\left(w_{0}, w_{1}\right)=1 \leqslant 1-0=\gamma(0)-\gamma(1)$ and $\forall_{m \in \mathbb{N}}\left\{U\left(w_{m}, w_{m+1}\right)=0 \leqslant \gamma\left(w_{m}\right)-\right.$ $\left.\gamma\left(w_{m+1}\right)\right\}$.

Case 2. If $x \in(0,1)$, then each dynamic process $\left(w_{m}: m \in\{0\} \cup \mathbb{N}\right)$ starting at $w_{0}=x$ is of the form $w_{m} \in T\left(w_{m-1}\right)=\left[0,(1 / 2) w_{m-1}\right]$ for $m \in \mathbb{N}$. Therefore, we have $\forall_{m \in\{0\} \cup \mathbb{N}}\{0<$ $\left.U\left(w_{m}, w_{m+1}\right)=w_{m}-w_{m+1}=\gamma\left(w_{m}\right)-\gamma\left(w_{m+1}\right)\right\}$.

Case 3. If $x=1$, then a dynamic process starting at $w_{0}=1$ is of the form $\forall_{m \in \mathbb{N}}\left\{w_{m}=1 \in\right.$ $\left.T\left(w_{m-1}\right)\right\}$ and $\forall_{m \in\{0\} \cup \mathbb{N}}\left\{U\left(w_{m}, w_{m+1}\right)=0 \leqslant \gamma\left(w_{m}\right)-\gamma\left(w_{m+1}\right)\right\}$.

Thus conditions (1.1) and (4.4) hold.

(b) We observe that

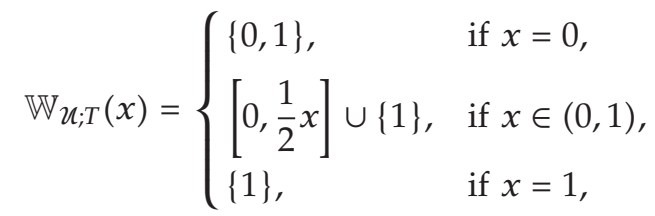

and $\mathbb{W}_{\mathcal{U} ; T}$ is $(\mathcal{U}, \Gamma)$-dissipative on $X$.

(c) For each $x \in X$, the set $\mathbb{W}_{\mathcal{U} ; T}(x)$ is closed.

(d) The assertions of Theorem 6.2( $\left.\mathrm{a}_{2}\right)$ hold.

(e) The map $\mathbb{W}_{\mathcal{U} ; T}$ is not closed.

(f) We have that $w=1 \in \operatorname{End}(T)$ and $\bigcap_{m \geqslant 0} \operatorname{cl}\left(\mathbb{W}_{\mathcal{U}_{;} T}\left(w_{m}\right)\right)=\{w\}$.

(g) The map $T$ is not lsc on $X$.

(h) The assumptions of Theorem 6.2 $\left(\mathrm{a}_{3}\right)$ do not hold.

Now, we present comparisons between our results and the well-known ones. The results of Kirk and Saliga [12] and Aubin and Siegel [1], concerning the existence of fixed points and endpoints of dissipative single-valued and set-valued dynamic systems, respectively, we may read as follows.

Theorem 7.14 (Kirk and Saliga [12, Theorem 1.1]). Let $(X, d)$ be a complete metric space and let $(X, T)$ be a single-valued closed dynamic system satisfying

$$
\forall_{x \in X}\{d(x, T(x)) \leqslant \varphi(x)-\varphi(T(x))\},
$$


where $\varphi: X \rightarrow \mathbb{R}$ is a map bounded from below. Then, for each $x \in X,\left\{T^{[m]}(x)\right\}$ converges to a fixed point of $T$.

Here, we may assume, without loss of generality, that $\varphi: X \rightarrow[0, \infty)$; in another case, we replace $\varphi$ by $\varphi_{0}=\varphi-\inf _{x \in X} \varphi(x)$.

Theorem 7.15 (Aubin and Siegel [1, Theorem 2.4]). Let $(X, d)$ be a complete metric space and let $(X, T)$ be a set-valued closed dynamic system satisfying

$$
\forall_{x \in X} \exists_{y \in T(x)}\{d(x, y) \leqslant \varphi(x)-\varphi(y)\}
$$

where $\varphi: X \rightarrow[0, \infty)$. Then, for each $x \in X$, there exists a dynamic process $\left(w_{m}: m \in\{0\} \cup \mathbb{N}\right)$ starting at $w_{0}=x$ of the system $(X, T)$ which converges to a fixed point of $T$.

Theorem 7.16 (Aubin-Siegel [1, Theorem 4.10]). Let $(X, d)$ be a complete metric space and let $(X, T)$ be a set-valued dynamic system satisfying

$$
\forall_{x \in X} \forall_{y \in T(x)}\{d(x, y) \leqslant \varphi(x)-\varphi(y)\}
$$

where $\varphi: X \rightarrow[0, \infty)$. Assume that $(a) T$ is $l s c ;$ or $(b) \mathbb{W}_{d ; T}$ is closed. Then $\operatorname{End}(T) \neq \emptyset$.

Remark 7.17. It is worth noticing that

(i) by Example 7.2(A), Theorem 5.3(b) when $q=1$ includes Theorems 7.14 and 7.15,

(ii) by Example 7.2(A) and Remark 5.1(i), Theorems 6.2( $\left(\mathrm{a}_{2}\right)$ and 6.2( $\left.\mathrm{a}_{3}\right)$ include Theorem 7.16.

(iii) the map $T$ defined in Example 7.7 satisfies the assumptions of Theorem 5.3(a) but does not satisfy the assumptions of Theorems 7.14, 7.15, and 7.16(a). Indeed, first let us observe that $T$ is closed (and lsc as continuous). Next, suppose that the map $T$ satisfies the assumptions of Theorems 7.14, 7.15, and 7.16(a). Then there exists a map $\varphi: X \rightarrow[0, \infty)$ such that the condition $\forall_{x \in X}\{d(x, T(x)) \leqslant \varphi(x)-\varphi(T(x))\}$ holds. Then, in particular, for $x=0$, we have that $0<d(x, T(x))=d(0,1)=1 \leqslant$ $\varphi(0)-\varphi(1)$, which means that $\varphi(1)<\varphi(0)$. On the other hand, for $x=1$, we get $0<d(x, T(x))=d(1,0)=1 \leqslant \varphi(1)-\varphi(0)$, which means that $\varphi(0)<\varphi(1)$. This is absurd.

(iv) The map $T$ defined in Example 7.13 satisfies the assumptions of Theorem 6.2( $\left(\mathrm{a}_{2}\right)$ but does not satisfy the assumptions of Theorems 7.16(a) and 7.16(b). This follows from (e) and (g).

\section{References}

[1] J.-P. Aubin and J. Siegel, "Fixed points and stationary points of dissipative multivalued maps," Proceedings of the American Mathematical Society, vol. 78, no. 3, pp. 391-398, 1980.

[2] J.-P. Aubin and I. Ekeland, Applied Nonlinear Analysis, Pure and Applied Mathematics, John Wiley \& Sons, New York, NY, USA, 1984.

[3] J.-P Aubin and H. Frankowska, Set-Valued Analysis, vol. 2 of Systems \& Control: Foundations $\mathcal{E}$ Applications, Birkhäuser, Boston, Mass, USA, 1990. 
[4] G. X.-Z. Yuan, KKM Theory and Applications in Nonlinear Analysis, vol. 218 of Monographs and Textbooks in Pure and Applied Mathematics, Marcel Dekker, New York, NY, USA, 1999.

[5] E. Tarafdar and R. Vyborny, "A generalized (multivalued) contraction mapping principle," in Research Report of Pure Mathematics, vol. 54, The University of Queensland, Brisbane, Australia, 1976.

[6] E. Tarafdar and G. X.-Z. Yuan, "Set-valued topological contractions," Applied Mathematics Letters, vol. 8, no. 6, pp. 79-81, 1995.

[7] J. Caristi, "Fixed point theorems for mappings satisfying inwardness conditions," Transactions of the American Mathematical Society, vol. 215, pp. 241-251, 1976.

[8] I. Ekeland, "On the variational principle," Journal of Mathematical Analysis and Applications, vol. 47, pp. 324-353, 1974.

[9] W. A. Kirk, "Fixed points of asymptotic contractions," Journal of Mathematical Analysis and Applications, vol. 277, no. 2, pp. 645-650, 2003.

[10] S. Banach, "Sur les opérations dans les ensembles abstraits et leurs applications aux équations intégrales," Fundamenta Mathematicae, vol. 3, pp. 133-181, 1922.

[11] M. Edelstein, "On fixed and periodic points under contractive mappings," Journal of the London Mathematical Society, vol. 37, pp. 74-79, 1962

[12] W. A. Kirk and L. M. Saliga, "The Brézis-Browder order principle and extensions of Caristi's theorem," Nonlinear Analysis: Theory, Methods \& Applications, vol. 47, no. 4, pp. 2765-2778, 2001.

[13] C. Ding and S. B. Nadler Jr., "The periodic points and the invariant set of an $\epsilon$-contractive map," Applied Mathematics Letters, vol. 15, no. 7, pp. 793-801, 2002.

[14] S. B. Nadler Jr., "Periodic points of multi-valued $\varepsilon$-contractive maps," Topological Methods in Nonlinear Analysis, vol. 22, no. 2, pp. 399-409, 2003.

[15] E. Tarafdar and G. X.-Z. Yuan, "The set-valued dynamic system and its applications to Pareto optima," Acta Applicandae Mathematicae, vol. 46, no. 1, pp. 93-106, 1997.

[16] E. Tarafdar and M. S. R. Chowdhury, Topological Methods for Set-Valued Nonlinear Analysis, World Scientific, Hackensack, NJ, USA, 2008.

[17] J. C. Willems, "Dissipative dynamical systems," Archive for Rational Mechanics and Analysis, vol. 45, pp. 321-351, 1972.

[18] W. Zangwill, Nonlinear Programming, Prentice-Hall, Englewood Cliffs, NJ, USA, 1969.

[19] M. Justman, "Iterative processes with "nucleolar" restrictions," International Journal of Game Theory, vol. 6, no. 4, pp. 189-212, 1977.

[20] M. Maschler and B. Peleg, "Stable sets and stable points of set-valued dynamic systems with applications to game theory," SIAM Journal on Control and Optimization, vol. 14, no. 6, pp. 985-995, 1976.

[21] A. Petruşel and A. Sîntămărian, "Single-valued and multi-valued Caristi type operators," Publicationes Mathematicae Debrecen, vol. 60, no. 1-2, pp. 167-177, 2002.

[22] K. Włodarczyk, R. Plebaniak, and M. Doliński, "Cone uniform, cone locally convex and cone metric spaces, endpoints, set-valued dynamic systems and quasi-asymptotic contractions," Nonlinear Analysis: Theory, Methods \& Applications, vol. 71, no. 10, pp. 5022-5031, 2009.

[23] K. Włodarczyk, R. Plebaniak, and C. Obczyński, "Convergence theorems, best approximation and best proximity for set-valued dynamic systems of relatively quasi-asymptotic contractions in cone uniform spaces," Nonlinear Analysis: Theory, Methods \& Applications, vol. 72, no. 2, pp. 794-805, 2010.

[24] K. Deimling, Nonlinear Functional Analysis, Springer, Berlin, Germany, 1985.

[25] C. Berge, Topological Spaces, Oliver \& Boyd, Edinburgh, UK, 1963.

[26] E. Klein and A. C. Thompson, Theory of Correspondences: Including Applications to Mathematical Economics, Canadian Mathematical Society Series of Monographs and Advanced Texts, John Wiley \& Sons, New York, NY, USA, 1984.

[27] L.-G. Huang and X. Zhang, "Cone metric spaces and fixed point theorems of contractive mappings," Journal of Mathematical Analysis and Applications, vol. 332, no. 2, pp. 1468-1476, 2007. 